\title{
A detailed exploration of intermolecular interactions in 4-(4-dimethylaminobenzylideneamino)-N-(5-methyl-3-isoxazolyl) benzenesulfonamide and related Schiff bases: Crystal structure, spectral studies, DFT methods, Pixel energies and Hirshfeld surface analysis
}

\author{
Diego M. Gil ${ }^{\mathrm{a}, *, 1}$, Fernando F. Salomón ${ }^{\text {a }}$, G.A. Echeverría ${ }^{\mathrm{b}, 1}$, Oscar E. Piro ${ }^{\mathrm{b}, 1}$, Hiram Pérez ${ }^{\mathrm{c}, *}$, Aida Ben Altabef ${ }^{\mathrm{a}, *}$ \\ a INQUINOA (CONICET-UNT), Facultad de Bioquímica, Química y Farmacia, Universidad Nacional de Tucumán, San Lorenzo 456, T4000CAN San Miguel de Tucumán, Argentina \\ b Departamento de Física, Facultad de Ciencias Exactas, Universidad Nacional de La Plata y IFLP (CONICET, CCT-La Plata), C. C. 67, 1900 La Plata, Argentina \\ c Departamento de Química General e Inorgánica, Facultad de Química, Universidad de la Habana, Habana 10400, Cuba
}

\section{A R T I C L E I N F O}

\section{Article history:}

Received 24 April 2017

Received in revised form 26 May 2017

Accepted 29 May 2017

Available online 31 May 2017

\section{Keywords:}

Sulfa drugs

Schiff bases

Intermolecular interactions

Hirshfeld surfaces

Crystal structure

IR and Raman spectroscopy

Electronic spectra

\begin{abstract}
A B S T R A C T
The Schiff base of the title has been synthesized and its crystal structure determined by single-crystal X-ray diffraction. The compound was characterized by IR, Raman, ${ }^{1} \mathrm{H}$ NMR, ${ }^{13} \mathrm{C}$ NMR and electronic absorption spectra. DFT calculations provide the quantum chemical basis for the observed molecular conformation. A study of intermolecular interactions of the title compound is compared with seven other closely related structures and reveals that molecules in most of the compounds are linked by a cooperative effect of strong and weak hydrogen bonds, $\mathrm{C}-\mathrm{H}^{\cdots} \pi$, and $\pi \cdots \pi$ stacking interactions, and also lp ${ }^{\cdots} \pi$ contacts. Lattice energy calculations indicate that the dispersion component is the major contribution, with the coulombic term playing a significant role in the total energy. Interaction energies for molecular pairs involving $\mathrm{N}-\mathrm{H} \cdots \mathrm{N}$ bonds indicate a dominant contribution to packing stabilization coming from coulomb component. Hirshfeld surfaces and 2D-fingerprint plots allowed us to visualize different intermolecular contacts and its relative contributions to total surface in each compound. The analysis of electrostatic potential (ESP) maps correlates well with the computed energies providing evidences on the dominant electrostatic nature of $\mathrm{N}-\mathrm{H} \cdots \mathrm{N}$ and $\mathrm{N}-\mathrm{H} \cdots \mathrm{O}$ interactions.
\end{abstract}

@ 2017 Published by Elsevier B.V.

\section{Introduction}

Sulfonamides are considered potent antimicrobial agents [1]. Nowadays there are some problems associated with the occurrence of drug-resistant microbial strains and the demand for new and better antibacterial compounds makes relevant the research on this field and the search for new antimicrobial agents. Sulfonamides can be used for the treatment of different infections including chancroid, nocardiosis and infections in the urinary tract caused by some microorganism like $E$. coli and others. These compounds can exhibit a wide variety of pharmacological activities such as anti-tumor, anti-viral, anti-fungal, anticarbonic anhydrase, diuretic, among others [2-5].

Sulfamethoxazole is a well-known antibacterial sulfa drug and the presence of the amino group in this compound leads the formation of

\footnotetext{
* Corresponding authors at: Instituto de Química Física, Facultad de Bioquímica, Química y Farmacia, Universidad Nacional de Tucumán, San Lorenzo 456, T4000CAN San Miguel de Tucumán, Argentina.

E-mail addresses: dmgil@fbqf.unt.edu.ar (D.M. Gil), alinaca@infomed.sld.cu (H. Pérez), altabef@fbqf.unt.edu.ar (A.B. Altabef).

${ }^{1}$ Members of the Research Career of CONICET.
}

Schiff bases by condensation with aldehydes. Schiff bases provide more potential sites for chemical and biological activities of different compounds. Schiff bases are characterized by the azomethine functional group $(-\mathrm{RC}=\mathrm{N}-)$ and have a wide range of biological activities, including anti-bacterial, anti-fungal, anti-malarial, anti-viral, anti-proliferative and anti-inflammatory properties [6-8]. Schiff bases are also of interest in industrial fields [9] and as corrosion inhibitors [10], thermo-stable materials [11], as well as, powerful ligands in the formation of coordination compounds [12]. Recently, Mondal and co-workers have reported the synthesis and structural characterization of Schiff bases derived from sulfamethoxazole and substituted salicylaldehydes [13]. These compounds exhibit antimicrobial activity against several Gram-positive and Gram-negative bacteria.

In continuation of our interest on Schiff bases [14], in the present work we report the synthesis, crystal structure and spectroscopic study of the Schiff base 4-(4-dimethyl aminobenzylideneamino)-N(5-methyl-3-isoxazolyl)benzenesulfonamide (for short, BA-SMX). The substance was characterized by IR, Raman, UV-Vis, ${ }^{1} \mathrm{H}$ and ${ }^{13} \mathrm{C}$ NMR, electronic spectra and single-crystal X-ray diffraction. The measurements were complemented with quantum chemical calculations based on DFT methods. Lattice energies and pair-wise energy 
interaction of different molecules have been calculated to determine the different energy components contributing to crystal stabilization $[15,16]$ for BA-SMX and seven other related structures. Hirshfeld surface (HS) based tools such as $d_{\text {norm }}$, shape index and curvedness surface properties [17-20] have been used to explore the packing modes and visualize the intermolecular interactions. Full and decomposed 2Dfingerprint plots have been derived from HS to determine the relative contribution of each type of interaction [21,22]. Furthermore, electrostatic potential (ESP) maps $[23,24]$ have been calculated to visualize and estimate the electrostatic nature of interactions, which are important to estimate how the molecules align and link to biological receptors. Finally, the Natural Bond Orbital (NBO) analysis was performed and the donor-acceptor energies were calculated by the second-order perturbation theory. This study was employed to calculate donor interaction energies, which provide an appropriate basis for understanding charge transfer or conjugative interactions in the analyzed molecular system.

\section{Experimental}

\subsection{Synthesis}

The Schiff base BA-SMX was synthesized by condensation of sulfamethoxazole (SMX) and 4-(dimethylamino)benzaldehyde (BA). An ethanol solution of SMX ( $1 \mathrm{mmol}$ ) was mixed with $1 \mathrm{mmol}$ of BA in ethanol. The mixture was stirred and refluxed at $80^{\circ} \mathrm{C}$ during $12 \mathrm{~h}$ and the progress was followed by TLC. On completion, the reaction mixture was cooled and the yellow solid formed was isolated by filtration and washed with cool ethanol. The solid was re-crystallized from hot ethanol. Suitable crystals for XRD measurements were obtained by slow evaporation of a methanol solution of BA-SMX (See Scheme 1)

Compound 1:

4-(4-dimethylaminobenzylideneamino)-N-(5-methyl-3-isoxazolyl) benzenesulfonamide (BA-SMX). Yellow crystalline solid; yield: 56\%; melting point: $228{ }^{\circ} \mathrm{C} ;{ }^{1} \mathrm{H}$ NMR $\left(\mathrm{CDCl}_{3}, \delta \mathrm{ppm}\right): 2.37$ (d, $\left.3 \mathrm{H}\right), 3.07$ $(\mathrm{s}, 6 \mathrm{H}), 6.25(\mathrm{~d}, 1 \mathrm{H}), 6.75(\mathrm{~d}, 2 \mathrm{H}), 7.19(\mathrm{~d}, 2 \mathrm{H}), 7.74(\mathrm{~d}, 2 \mathrm{H}), 7.81$ $(\mathrm{d}, 2 \mathrm{H}), 8.23(\mathrm{~s}, 1 \mathrm{H}) .{ }^{13} \mathrm{C}$ NMR $\left(\mathrm{CDCl}_{3}, \delta \mathrm{ppm}\right): 12.61$ (C-10), 40.05 (C-19), 95.53 (C-8), 111.53 (C-16), 121.67 (C-5), 124.11 (C-12), 128.49 (C-6), 131.12 (C-17), 134.43 (C-1), 153.12 (C-15), 157.57 (C-4, C-7), 162.23 (C-11), 171.08 (C-9).

\subsection{Instrumentation}

The melting point was determined by Differential Scanning Calorimetry (DSC) on a Perkin Elmer Pyris DSC 6 calorimeter. The ${ }^{1} \mathrm{H}(200 \mathrm{MHz})$ and ${ }^{13} \mathrm{C}(50.3 \mathrm{MHz})$ NMR spectra were recorded on a Varian Unity 200 spectrometer in $\mathrm{CDCl}_{3}$ using tetramethylsilane (TMS) as internal reference. The solid state IR absorption spectra were measured on an FTIR Perkin Elmer GX1 in the $4000-400 \mathrm{~cm}^{-1}$ range. The Raman dispersion spectra were recorded using a Thermoscientific DXR Raman microscope. The UV-Visible spectra were measured with a Beckman/DU 7500 spectrophotometer in the spectral region of $200-800 \mathrm{~nm}$ on a sample dissolved in acetonitrile at a concentration of $10^{-5} \mathrm{M}$.

\subsection{X-ray Diffraction Data}

The measurements were performed on an Oxford Xcalibur, Eos, Gemini CCD diffractometer with graphite-monochromated $\mathrm{CuK} \alpha$ $(\lambda=1.54184 \AA)$ radiation. X-ray diffraction intensities were collected ( $\omega$ scans with $\vartheta$ and $\kappa$-offsets), integrated and scaled with CrysAlisPro [25] suite of programs. The unit cell parameters were obtained by least-squares refinement (based on the angular settings for all collected reflections with intensities larger than seven times the standard deviation of measurement errors) using CrysAlisPro. Data were corrected empirically for absorption employing the multi-scan method implemented in CrysAlisPro.

The structure was solved by the 'intrinsic phasing' method implemented in SHELXT program [26]. The initial molecular model was refined with atomic anisotropic displacement parameters by full-matrix least-squares procedure with SHELXL of the SHELX package [26]. All $\mathrm{H}$-atoms were found in a difference-Fourier map. All hydrogen atoms, but the ones of the methyl groups, were refined at their found positions with isotropic displacement parameters. The methyl H-atoms were refined with the riding model, as rigid groups allowed to rotate around their corresponding $\mathrm{C}-\mathrm{C}$ and $\mathrm{N}-\mathrm{C}$ bonds such as to maximize the sum of the observed residual electron density at their calculated positions. Crystal data and structure refinement results are summarized in Table 1.

Geometric parameters of $\pi$-stacking interactions were calculated with PLATON for Windows Taskbar v1.17 [27] and the molecular structure was plotted using MERCURY [28].

Full crystallographic data have been deposited with the Cambridge Crystallographic Data Centre (CCDC 1543530).

\subsection{Computational Methods}

Quantum chemical calculations have been performed using the Gaussian 03 program [29]. Geometry optimizations and vibration frequency calculations were carried out with the Density Functional Theory (DFT) using the hybrid functional with non-local exchange due to Becke (B3) and the correlation functional due to Lee-Yang-Parr [30, 31]. The calculations were carried out using $6-311++G(d, p)$ basis set. The potential energy distribution (PED) was calculated using VEDA4 program [32,33]. Electronic transitions were calculated within the Time-Dependent Density Functional Theory (TD-DFT) [34] in gas phase and taking into account implicitly the solvent effect (acetonitrile) at B3LYP/6-311 ++G(d,p) level of theory. The Natural Bond Orbital (NBO) analysis for the compound under study was performed at $\mathrm{B} 3 \mathrm{LYP} / 6-311++\mathrm{G}(\mathrm{d}, \mathrm{p})$ approximation using the NBO 3.1 program implemented in the Gaussian 03 package [29]. The Gauge-including atomic orbital (GIAO) method [35] was used to calculate the ${ }^{1} \mathrm{H}$ and ${ }^{13} \mathrm{C}$ chemical shifts at B3LYP/6-311 $+\mathrm{G}(2 \mathrm{~d}, \mathrm{p})$ approximation.

\subsection{Lattice and Interaction Energies}

For each crystal under consideration lattice energy and intermolecular interaction energies for specific molecular pairs were calculated using the CLP (Coulomb-London-Pauli) approach implemented in the<smiles>CCC(C)(C)N(C)c1ccc(/C=N/c2ccc(S(=O)(=O)Nc3cc(C)on3)cc2)cc1</smiles>

SMX

BA

BA-SMX

Scheme 1. Synthesis of 4-(4-dimethylaminobenzylideneamino)-N-(5-methyl-3-isoxazolyl)benzenesulfonamide. 
Table 1

Crystal data and structure refinement for BA-SMX (compound $\mathbf{1}$ ).

\begin{tabular}{|c|c|}
\hline Empirical formula & $\mathrm{C}_{19} \mathrm{H}_{20} \mathrm{~N}_{4} \mathrm{O}_{3} \mathrm{~S}$ \\
\hline Formula weight & 384.45 \\
\hline Temperature/K & $293(2)[2]$ \\
\hline Crystal system & monoclinic \\
\hline Space group & $\mathrm{P} 21 / \mathrm{n}$ \\
\hline Unit cell dimensions & $\begin{array}{l}a=15.7553(5) \AA \\
b=6.9061(2) \AA \\
c=18.6278(5) \AA \\
\beta=110.789(3)^{\circ}\end{array}$ \\
\hline Volume $/ \AA^{3}$ & $1894.9(1)$ \\
\hline Z & 4 \\
\hline$\rho$ calc. $/ \mathrm{mg} \mathrm{mm}^{-3}$ & 1.348 \\
\hline$\mu / \mathrm{mm}^{-1}$ & 1.751 \\
\hline$F(000)$ & 808 \\
\hline Crystal size $/ \mathrm{mm}^{3}$ & $0.295 \times 0.158 \times 0.079$ \\
\hline Theta range for data collection & 3.17 to $72.52^{\circ}$ \\
\hline Index ranges & $\begin{array}{l}-19 \leq h \leq 17 \\
-8 \leq k \leq 5 \\
-18 \leq l \leq 22\end{array}$ \\
\hline Reflections collected & 7547 \\
\hline Independent reflections & $3690[R($ int $)=0.0221]$ \\
\hline Data/restraints/parameters & $3690 / 0 / 291$ \\
\hline Completeness to theta & $67.684(100 \%)$ \\
\hline Goodness-of-fit on $F^{2}$ & 1.058 \\
\hline Final $R$ indexes $^{\mathrm{a}}[I>2 \sigma(I)]$ & $\begin{array}{l}R 1=0.0392 \\
\mathrm{w} R 2=0.1130\end{array}$ \\
\hline Largest diff. peak/hole/e $\AA^{-3}$ & $0.349 /-0.228$ \\
\hline
\end{tabular}

${ }^{\mathrm{a}} R_{1}=\Sigma \|\left|F_{o}\right|-\left|F_{c}\right| / \Sigma\left|F_{o}\right|, \mathrm{w} R_{2}=\left[\Sigma w\left(\left|F_{o}\right|^{2}-\left|F_{c}\right|^{2}\right)^{2} / \Sigma w\left(\left|F_{o}\right|^{2}\right)^{2}\right]^{1 / 2}$.

PIXEL program package, [36,37] which enables partitioning of the total energy into their coulombic, polarization, dispersion and repulsion contributions.

\subsection{Hirshfeld Surface Calculations}

Hirshfeld surfaces and the associated 2D-fingerprint plots [38-40] are generated using CrystalExplorer3.0 software [41]. The normalized contact distance $\left(d_{\text {norm }}\right)$ enables the identification of the regions of particular importance to the intermolecular interactions, being $d_{\text {norm }}$ a symmetric function of distances to the surface from nuclei inside and outside the Hirshfeld surface $\left(d_{\mathrm{i}}\right.$ and $d_{\mathrm{e}}$, respectively), relative to their respective van der Waals radii. Hirshfeld surfaces for title structure were also mapped with shape index and curvedness properties. The 2D-fingerprint plot provides decomposition of Hirshfeld surfaces into relative contribution of different intermolecular interactions present in crystal structure. A color scale of red (shorter than vdW separation)white (equal to vdW separation)-blue (longer than vdW separation) is used to visualize the intermolecular contacts. The $3 \mathrm{D} d_{\text {norm }}$ surfaces are mapped over a fixed color scale of -0.050 (red) to 0.140 (blue), shape index mapped in the color range of -1.0 a.u. (concave) to 1.0 a.u. (convex) $\AA$, and curvedness in the range of -4.0 a.u. (flat) to 4.0 a.u. (singular) $\AA$. The $2 \mathrm{D}$-fingerprint plots are displayed by using the translated 0.6-2.8 Å range, and including reciprocal contacts. Decomposed electrostatic potentials were mapped on the Hirshfeld surfaces on $0.008 \mathrm{e}^{-3}$ isosurfaces over the range -0.06 a.u (red), through 0 (white), to 0.06 a.u (blue) [42], at the Hartree-Fock theory and 6$31 \mathrm{G}(\mathrm{d}) / \mathrm{cc}-\rho \mathrm{VDZ}$ basis sets using the TONTO program[43] integrated into CrystalExplorer.

\section{Results and Discussion}

\subsection{Description of Molecular and Crystal Structure}

Fig. 1 shows an ORTEP [44] drawing of the molecular structure of BASMX determined by X-ray diffraction methods (a) together with the computed molecular structure (b) at B3LYP/6-311 ++G(d,p) approximation. Selected bond lengths, angles and torsion angles are listed in
Table 2. The $\mathrm{C} 1$-S bond length of 1.760(2) A is in accordance with a normal single-bond value and matches well with those observed in other sulfonamides [45-48]. The S-N bond length of 1.637(2) A indicates a double-bond character [49]. The $\mathrm{S}-\mathrm{O}$ bond distances are similar to those found in similar structures [45-48]. The C18-N4-C15 $\left[121.0(2)^{\circ}\right]$ and $\mathrm{C} 19-\mathrm{N} 4-\mathrm{C} 15\left[121.5(2)^{\circ}\right]$ bond angles around $120^{\circ}$ are consistent with the $s p^{2}$ hybrid character of $\mathrm{N} 4$ atom. These distances do not vary significantly in sulfonamides structures despite the different intermolecular interaction pattern observed. The bond lengths and angles in benzene rings have characteristic values as expected for a resonant structure. The angular disposition of bonds around the $S$ atom deviates significantly from that of a regular tetrahedron. The largest deviation is in the angle $\mathrm{O} 1-\mathrm{S}-\mathrm{O} 2\left[120.71(9)^{\circ}\right]$ and the smallest value is observed in the angle $\mathrm{N} 2-\mathrm{S}-\mathrm{O} 2\left[107.67(9)^{\circ}\right]$. These results are in agreement with the non-tetrahedral arrangement commonly observed in sulfonamides [45-48,50,51]. The $\mathrm{C}=\mathrm{N}$ bond length of $1.277(2) \AA \AA$ is consistent with a $\mathrm{C}=\mathrm{N}$ double bonding.

The group of atoms involving the R1-ring (C12-C17) and the azomethine $\mathrm{C} 11=\mathrm{N} 1$ double bond is nearly planar, with the largest deviations from the best plane being - 0.0545(1) Å for C11 atom. The R2ring $(\mathrm{C} 1-\mathrm{C} 6)$ is significantly twisted around $\mathrm{N} 1-\mathrm{C} 4$ bond as reflected by the $\mathrm{C} 11-\mathrm{N} 1-\mathrm{C} 4-\mathrm{C} 3$ torsion angle of $136.4(2)^{\circ}$. The N4/C18-C19 fragment and R1-ring plane are nearly coplanar, showing C18-N4-C15-C14 and C19-N4-C15-C14 torsion angles of $-177.6(2)^{\circ}$ and $-14.6(3)^{\circ}$, respectively. The dihedral angle between the isoxazole (N3-03/C7-C9) and aminophenyl (C1-C6) mean planes is $83.2(1)^{\circ}$. These results are similar to those obtained for similar structures [14], and the overall molecule adopts an $E$ conformation related to the azomethine group with $\mathrm{C} 12-\mathrm{C} 11-\mathrm{N} 1-\mathrm{C} 4$ torsion angle of $169.2(2)^{\circ}$. As can be seen in Table 2 , a very good agreement between calculated and experimental geometrical parameters has been observed.

2-Hydroxybenzaldehyde Schiff bases have generally a planar structure due to the existence of intramolecular $\mathrm{N}-\mathrm{H} \cdots \mathrm{O}$ hydrogen bonds [45-48]. However, this type of interaction is absent in the structure reported here, being $57.8(1)^{\circ}$ the dihedral angle between the mean planes of two benzene rings.

\subsection{Lattice Energies, Structural Motifs and Interaction Energies}

Packing diagrams for title structure (hence forth $\mathbf{1}$ ) are depicted in Fig. 2. Geometry of relevant intermolecular hydrogen bonds is listed in Table 3 (Column 4). In order to compare the packing mode of 1 with similar structures, a search of the CSD crystal structure database for benzylideneamino-N-benzenesulfonamide moiety was undertaken yielding seven hits, four of which are substituted $R$-benzylideneamino$\mathrm{N}$-(5-methyl-3-isoxazolyl)benzenesulfonamide derivatives where $R=$ 2-hydroxy-3,5-dichloro, 2-hydroxy, 2-hydroxy-3-metoxy and 2-hydroxy-5-methylas substituents on the benzene ring. These four structures identified here by MULMA [13], POPJUA [48], SADHEM [52] and OKEWOS [53] refcodes, respectively, are closest to compound $\mathbf{1}$ with $R=$ 4-dimethylamine substituent.

Another two related structures are substituted $R$-benzylideneamino$\mathrm{N}$-(acetyl)benzensulfonamidemonohydrates presenting $R=2$-hydroxy(CIKJAI) [54] and 5-bromo-2-hydroxy (CIKJIQ) [54] substituents. Finally, with the purpose of evaluating energetically the role of substituents on the packing modes in this type of Schiff bases, the unsubstituted $4-\{[4-$

(dimethylamino)benzylidene]amino\}benzenesulfonamide (SODGID) [55] was included in our comparative study.

Lattice energy calculations for all the compounds are listed in Table 3, showing that the dispersion energy $\left(\mathrm{E}_{\mathrm{disp}}\right)$ is the major contribution towards the crystal stabilization for all compounds as generally expected for organic species. In compound $\mathbf{1}$ and the four related structures all presenting the aromatic isoxazole ring, the dispersive energy is in the range $190-229 \mathrm{~kJ} \mathrm{~mol}^{-1}$, which represents percentages varying from 


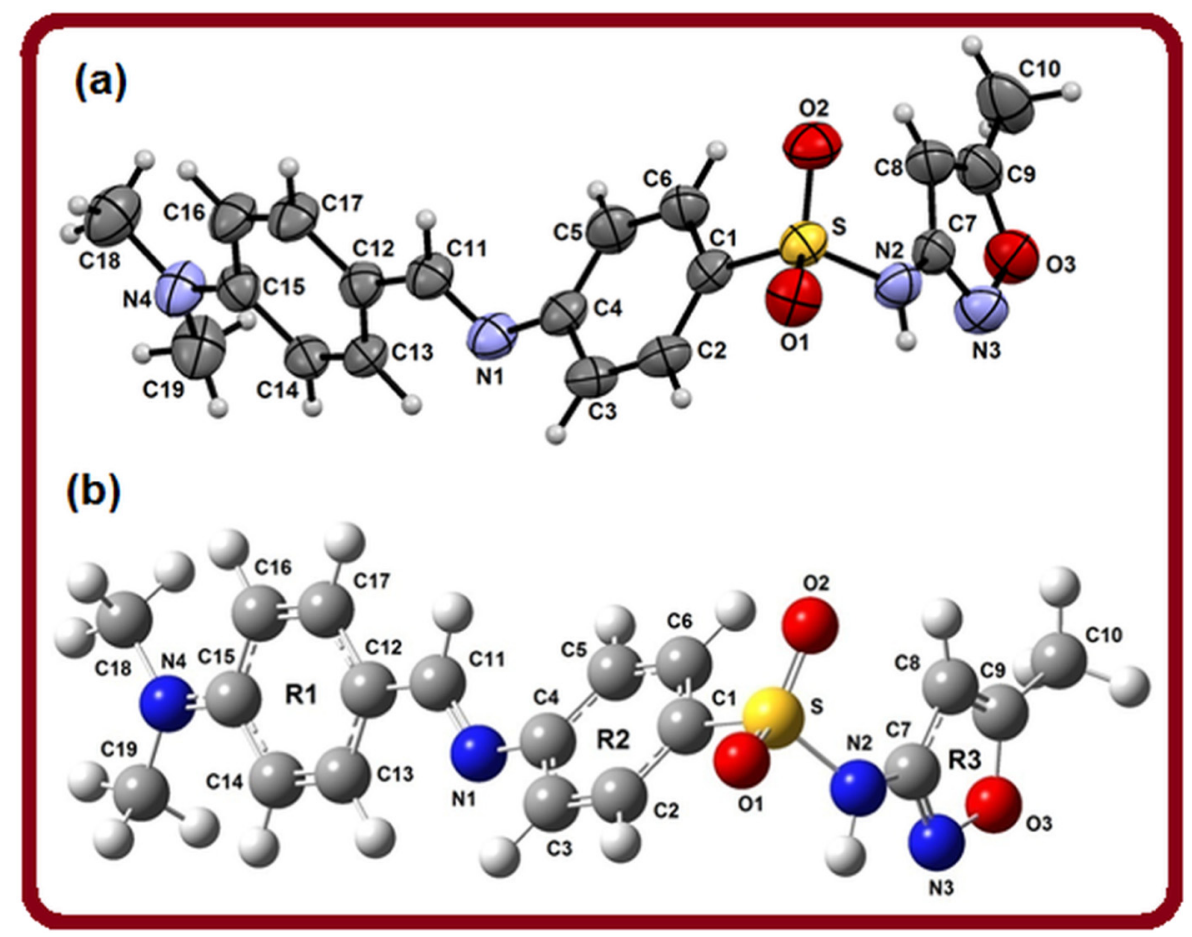

Fig. 1. (a) X-ray and (b) computed molecular structures of Schiff base BA-SMX. Displacement ellipsoids in (a) are plotted at $30 \%$ probability level.

$57.7 \%$ in POPJUA to $60.0 \%$ in OKEWOS with respect to the total cohesive energy. By the other hand, lower and similar dispersion energies in CIKJAI $\left(-102.7 \mathrm{~kJ} \mathrm{~mol}^{-1}\right)$ and CIKJIQ $\left(-110.5 \mathrm{~kJ} \mathrm{~mol}^{-1}\right)$ compounds showing the alkyl acetate substituent represent contributions of 44.9 and $47.9 \%$, respectively, which can be attributed to the structural similarity (based on lattice parameters and space group) between these two structures. It is interesting to note an intermediate contribution of $54.1 \%$ for dispersion energy in unsubstituted SODGID structure. These results allow suggesting that the nature of substituents at the amide

Table 2

Selected bond lengths $(\AA)$, angles and dihedral angles $\left({ }^{\circ}\right)$ observed and calculated for the Schiff base BA-SMX.

\begin{tabular}{lll}
\hline Parameters $^{\mathrm{a}}$ & Calculated B3LYP/6-311 $++\mathrm{G}(\mathrm{d}, \mathrm{p})$ & Experimental $^{\mathrm{b}}$ \\
\hline Bond lengths $(\AA)$ & & \\
C1-S & 1.789 & $1.760(2)$ \\
S-O1 & 1.457 & $1.426(1)$ \\
S-O2 & 1.458 & $1.425(1)$ \\
S-N2 & 1.708 & $1.637(2)$ \\
C11-N1 & 1.283 & $1.277(2)$ \\
C7-N2 & 1.398 & $1.397(3)$ \\
C4-N1 & 1.395 & $1.400(3)$ \\
Angles $\left(^{\circ}\right)$ & & \\
C12-C11-N1 & 123.3 & $124.1(2)$ \\
C11-N1-C4 & 120.4 & $118.7(2)$ \\
N1-C4-C5 & 122.9 & $121.2(2)$ \\
C1-S-O1 & 108.5 & $108.7(9)$ \\
C1-S-O2 & 108.2 & $108.24(8)$ \\
C1-S-N2 & 107.3 & $106.44(8)$ \\
O2-S-N2 & 105.7 & $107.67(9)$ \\
O1-S-N2 & 103.5 & $104.22(9)$ \\
S-N2-C7 & 122.5 & $123.2(1)$ \\
Torsional angles $\left({ }^{\circ}\right)$ & & \\
C17-C12-C11-N1 & 178.1 & $177.4(2)$ \\
C12-C11-N1-C4 & 176.4 & $169.2(2)$ \\
C11-N1-C4-C5 & -43.52 & $-47.6(3)$ \\
C1-S-N2-C7 & 65.09 & $65.7(2)$ \\
\hline a & &
\end{tabular}

\footnotetext{
a See Fig. 1 for atoms numbering scheme.
}

b This work. fragment controls the energetic components, and consequently the crystal stabilization in the series of eight analyzed structures.

Intermolecular interaction energies for all eight structures are given in Table 4. Different molecular pairs were selected involving the main intermolecular interactions in crystals. The crystal packing of compound 1 exhibits an interesting intermolecular pattern characterized by different structural motifs, being most relevant the formation of centre-symmetric dimers via strong $\mathrm{N} 2-\mathrm{H} 2 \cdots \mathrm{N} 3$ hydrogen bonds generating a $R_{2}^{2}$ (8) ring (motif I, Fig. 2a) with the highest interaction energy of $-68.0 \mathrm{~kJ} \mathrm{~mol}^{-1}$. We observe the same motif in three related structures (MULMA, POPJUA and OKEWOS), while the existence of centre-symmetric dimers through $\mathrm{N}-\mathrm{H} \cdots \mathrm{N}$ hydrogen bonds generates $R_{2}^{2}(16)$ rings in SODGID with highest total energy of $-103.7 \mathrm{~kJ} \mathrm{~mol}^{-1}$. In addition, the contribution from coulombic effect towards the total stabilization is dominant (53\%) for motif I in structure 1, and a similar energetic behavior is observed for the four related structures previously mentioned, the coulombic term ranging from $45.1 \%$ in SODGID to $53.4 \%$ in POPJUA. In SADHEM and monohydrate (CIKJAI and CIKJIQ) structures, $\mathrm{N}-\mathrm{H} \cdots \mathrm{N}$ hydrogen bonds were not observed, and instead the strong $\mathrm{N}-\mathrm{H} \cdots \mathrm{O}$ hydrogen bonds are involved in molecular pairs with highest interaction energy. In CIKJAI and CIKJIQ the coulombic contribution is dominant for $\mathrm{N}-\mathrm{H} \cdots \mathrm{O}$ motifs formed by water oxygen, representing $60 \%$ and $61 \%$ of the total stabilization energy, respectively. Although the dispersion component is largest for $\mathrm{N}-\mathrm{H} \cdots \mathrm{O}$ motifs in SADHEM, the electrostatic (coulomb + polarization) contribution of 58\% is comparable with the remaining structures.

In addition, other $\mathrm{H} \cdots \mathrm{N}$ contact due to $\mathrm{C} 14-\mathrm{H} 14 \cdots \mathrm{N} 1$ hydrogen bonds (motif II) provides the lowest energy $\left(-22.7 \mathrm{~kJ} \mathrm{~mol}^{-1}\right)$ in the crystal packing of 1 (Fig. 2a). Furthermore, the sulfonamide 01 oxygen atom acts as a bifurcated acceptor forming mutually perpendicular C18-H18A …01 (motif III) and C5-H5 …01 (motif IV) hydrogen bonds (Fig. $2 \mathrm{~b}$ ), the former through the $\mathrm{H} 18$ hydrogen atom from the methyl group, and the latest involving the $\mathrm{H} 5$ hydrogen atom from the phenyl ring of the sulfonamide group. The $\mathrm{C}-\mathrm{H} \cdots \mathrm{O}$ motifs are common in crystal packing of all eight structures, but the broad range of interaction energies (from - $13.6 \mathrm{~kJ} \mathrm{~mol}^{-1}$ in SADHEM to $-57.7 \mathrm{~kJ} \mathrm{~mol}^{-1}$ in POPJUA) to which are associated allows explaining 

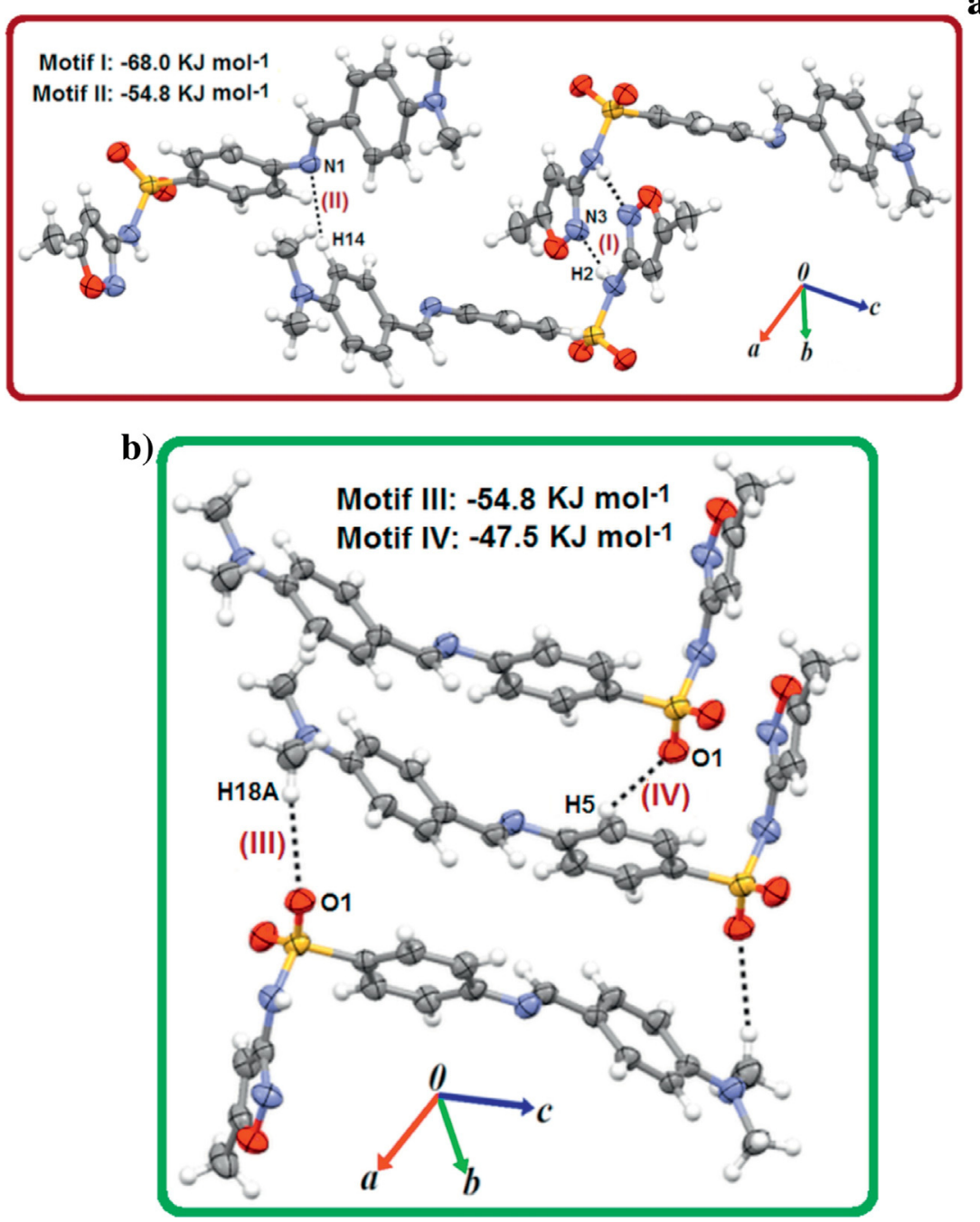

Fig. 2. Packing diagrams of compound 1 showing intermolecular (a) $\mathrm{N}-\mathrm{H} \cdots \mathrm{N}$ and $\mathrm{C}-\mathrm{H} \cdots \mathrm{N}$, (b) $\mathrm{C}-\mathrm{H} \cdots \mathrm{O}$ hydrogen bonds as dashed lines.

the formation of dimers with different graph-set patterns in these compounds. Thus, the occurrence of $R_{2}^{2}(18)$ rings through $\mathrm{C}-\mathrm{H} \cdots \mathrm{O}$ hydrogen bonds is observed in SADHEM, while $R_{1}^{2}(9)$ and $R_{2}^{2}$ (18) rings appear in POPJUA.

The supra-molecular assembly of $\mathbf{1}$ also includes the existence of $\pi \cdots \pi$ and $\mathrm{C}-\mathrm{H} \cdots \pi$ interactions. A significant $\mathrm{Cg} 1 \cdots \mathrm{Cg} 1$ interaction [inter-centroid distance $=3.9259 \AA$ ( 1 ), $\alpha$ (dihedral angle between the corresponding ring mean planes $)=0.00^{\circ}$, slippage $\left.=2.178 \AA\right]$ presents an offset arrangement involving the isoxazole ring ( $C g 1$ centroid) and represents a moderately strong $\pi$-stacking contact $\left(-33.3 \mathrm{~kJ} \mathrm{~mol}^{-1}\right)[56,57]$. Two different $\mathrm{C}-\mathrm{H} \cdots \pi$ contacts complete

Table 3

Lattice energies $\left(\mathrm{kJ} \mathrm{mol}^{-1}\right)$ partitioned into coulombic $\left(E_{\mathrm{coul}}\right)$, polarization $\left(E_{\mathrm{pol}}\right)$, dispersion $\left(E_{\mathrm{disp}}\right)$ and repulsion $\left(E_{\mathrm{rep}}\right)$ components for $\mathbf{1}$ and related compounds.

\begin{tabular}{llllll}
\hline Compound & $E_{\text {coul }}$ & $E_{\text {pol }}$ & $E_{\text {disp }}$ & $E_{\text {rep }}$ & $E_{\text {TOT }}$ \\
\hline $\mathbf{1}$ & -106.8 & -50.7 & -215.6 & 157.5 & -215.7 \\
MULMA & -115.5 & -48.3 & -224.8 & 159.2 & -229.3 \\
POPJUA & -94.9 & -44.2 & -190.0 & 130.9 & -198.2 \\
SADHEM & -96.1 & -42.2 & -204.0 & 134.9 & -207.4 \\
OKEWOS & -101.4 & -51.5 & -229.0 & 174.5 & -207.3 \\
SODGID & -118.5 & -54.4 & -203.5 & 156.2 & -220.2 \\
CIKJAI & -87.0 & -38.9 & -102.7 & 98.4 & -130.2 \\
CIKJIQ & -85.8 & -34.5 & -110.5 & 97.9 & -132.9
\end{tabular}

the 3D supra-molecular network. On one hand, the $\mathrm{C} 17-\mathrm{H} 17 \cdots \mathrm{Cg} 2$ interactions $(\mathrm{H} 17 \cdots \mathrm{Cg} 2$ distance $=2.93 \AA$, $\mathrm{C} 17-\mathrm{H} 17 \cdots \mathrm{Cg} 2$ angle $=$ $\left.130^{\circ}\right)$ involve the sulfonamide benzene ring ( $\mathrm{Cg} 2$ centroid) and operate along with the motif III in the same molecular pair $\left(-54.8 \mathrm{~kJ} \mathrm{~mol}^{-1}\right)$. By the other hand, $\mathrm{C} 3-\mathrm{H} 3 \cdots \mathrm{Cg} 3$ contacts $(\mathrm{H} 3 \cdots \mathrm{Cg} 3$ distance $=2.98 \AA$, $\mathrm{C} 3-\mathrm{H} 3 \cdots \mathrm{Cg} 3$ angle $\left.=140^{\circ}\right)$ involve the benzene ring $(\mathrm{Cg} 3$ centroid $)$ and act together with the motif IV $\left(-47.5 \mathrm{~kJ} \mathrm{~mol}^{-1}\right)$. The PIXEL energy results indicate that the $\pi$-stacking contacts in structure $\mathbf{1}$ are as important as the relatively stronger $\mathrm{N}-\mathrm{H} \cdots \mathrm{N}$ hydrogen bonds. This combined mode of cooperation of classical hydrogen bonding and $\pi$ stacking forces is also a feature observed in the crystal packing of all seven related structures (Table 4).

Interestingly, $\pi \cdots \pi$ and $\mathrm{C}-\mathrm{H} \cdots \pi$ interactions were not observed in the two monohydrates (CIKJAI and CIKJIQ). Instead, the Cg2 centroid of benzene ring for both structures is linked to the sulfonamide oxygen $\mathrm{O} 2$ atom through a significant lone-pair $1 \mathrm{p} \cdots \pi$ interaction [58], as reflected by a short $02 \cdots \mathrm{Cg} 2$ mean distance of $3.14 \AA$, and the mean angular distribution [deviation of the angle $\alpha$ ( $\alpha$ is the angle $\mathrm{S}-\mathrm{O} \cdots \mathrm{Cg}$ ) from $120^{\circ}$ ] of $13^{\circ}$. These $\mathrm{S}-\mathrm{O} \cdots \pi$ contacts were found with similar and notably higher interaction energies $\left(-58.8 \mathrm{~kJ} \mathrm{~mol}^{-1}\right.$ and $\left.-65.0 \mathrm{~kJ} \mathrm{~mol}^{-1}\right)$ than those for the strong $\mathrm{N}-\mathrm{H} \cdots 0$ hydrogen bonds $\left(-43.0 \mathrm{~kJ} \mathrm{~mol}^{-1}\right.$ and $-40.8 \mathrm{~kJ} \mathrm{~mol}^{-1}$ ). This type of interaction was also found in POPJUA structure with a lower average energy of $-29.0 \mathrm{~kJ} \mathrm{~mol}^{-1}$. These results confirm the relevant role of the $\pi$-stacking contacts in the series of studied Schiff bases. 
Table 4

Interaction energies $\left(E_{\mathrm{TOT}}\right)$ partitioned into coulombic, polarization, dispersion and repulsion contributions $\left(\mathrm{kJ}\right.$ mol $\left.{ }^{-1}\right)$ for various molecular pairs in $\mathbf{1}$ and related compounds.

\begin{tabular}{|c|c|c|c|c|c|c|c|c|c|}
\hline Compound & Symmetry & Involved interactions & $d(\mathrm{H} \cdots A), \angle D-\mathrm{H} \cdots A$ & Centroid distance & $E_{\text {coul }}$ & $E_{\mathrm{pol}}$ & $E_{\text {disp }}$ & $E_{\text {rep }}$ & $E_{\mathrm{TOT}}$ \\
\hline \multirow[t]{6}{*}{1} & $1-\mathrm{x}, 2-\mathrm{y}, 1-\mathrm{z}$ & $\mathrm{N} 2-\mathrm{H} 2 \cdots \mathrm{N} 3$ & $2.13(2), 172$ & 11.406 & -82.8 & -42.0 & -32.6 & 89.4 & -68.0 \\
\hline & $\begin{array}{l}1.5-x,-1 / 2+y \\
1 / 2-z\end{array}$ & $\mathrm{C} 14-\mathrm{H} 14 \cdots \mathrm{N} 1$ & $2.51(2), 156$ & 8.953 & -12.7 & -7.1 & -28.4 & 25.5 & -22.7 \\
\hline & $\mathrm{x},-1+\mathrm{y}, \mathrm{z}$ & $\mathrm{C} 5-\mathrm{H} 5 \cdots \mathrm{O} 1$ & $2.52(3), 130$ & 6.906 & -15.9 & -7.1 & -46.9 & 22.4 & -47.5 \\
\hline & $2-x, 1-y, 1-z$ & 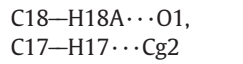 & $\begin{array}{l}2.665(1), 164 \\
2.93^{\mathrm{a}}\end{array}$ & 7.017 & -23.1 & -8.1 & -55.9 & 32.3 & -54.8 \\
\hline & $\mathrm{x}, 1+\mathrm{y}, \mathrm{z}$ & $\mathrm{C} 3-\mathrm{H} 3 \cdots \mathrm{Cg} 3$ & $2.98^{\mathrm{a}}$ & 6.906 & -15.9 & -7.1 & -46.9 & 22.4 & -47.5 \\
\hline & $1-\mathrm{x}, 1-\mathrm{y}, 1-\mathrm{z}$ & $\operatorname{Cg} 1 \cdots \operatorname{Cg} 1$ & $3.926(1)^{\mathrm{b}}$ & 8.834 & -10.8 & -4.0 & -29.5 & 11.0 & -33.3 \\
\hline \multirow[t]{3}{*}{ MULMA } & $1-\mathrm{x}, 1-\mathrm{y},-\mathrm{z}$ & $\mathrm{N} 2-\mathrm{H} 1 \cdots \mathrm{N} 3$ & $2.03(3), 175$ & 11.278 & -97.4 & -47.0 & -42.0 & 104.7 & -81.7 \\
\hline & $2-x,-1 / 2+y, 1 / 2-z$ & $\begin{array}{l}\mathrm{C} 13-\mathrm{H} 13 \cdots \mathrm{O} 3 \\
\mathrm{C} 15-\mathrm{H} 15 \cdots \mathrm{O} 1\end{array}$ & $\begin{array}{l}2.496(2), 162 \\
2.720(3), 154\end{array}$ & 8.701 & -23.7 & -8.2 & -30.6 & 22.4 & -40.1 \\
\hline & $2-\mathrm{x},-\mathrm{y},-\mathrm{z}$ & $\begin{array}{l}\mathrm{Cg} 2 \cdots \mathrm{Cg} 3 \\
\mathrm{C} 3-\mathrm{H} 3 \cdots \mathrm{Cg} 1\end{array}$ & $\begin{array}{l}3.8952(1)^{\mathrm{b}} \\
2.81^{\mathrm{a}}\end{array}$ & 5.159 & -24.5 & -11.8 & -110.4 & 69.0 & -77.7 \\
\hline \multirow[t]{11}{*}{ POPJUA } & $1-\mathrm{x},-\mathrm{y},-\mathrm{z}$ & N7-H7A $\cdots$ N5A & $2.12(2), 165$ & 11.070 & -91.3 & -48.3 & -31.4 & 100.0 & -71.0 \\
\hline & $-\mathrm{x}, 1-\mathrm{y},-\mathrm{z}$ & $\begin{array}{l}\mathrm{C} 12 \mathrm{~A}-\mathrm{H} 12 \mathrm{~A} \cdots \mathrm{O} 25 \mathrm{~A} \\
\mathrm{C} 2-\mathrm{H} 2 \mathrm{~A} \cdots \mathrm{O} 25 \mathrm{~A}\end{array}$ & $\begin{array}{l}2.70(2), 139 \\
2.61(2), 146\end{array}$ & 6.300 & -32.7 & -13.8 & -49.7 & 38.6 & -57.7 \\
\hline & $\mathrm{x}, 1+\mathrm{y},-1+\mathrm{z}$ & 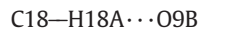 & $2.65(2), 157$ & 7.172 & -22.6 & -8.2 & -31.4 & 17.6 & -44.5 \\
\hline & $\begin{array}{l}-1+\mathrm{x}, 1+\mathrm{y} \\
-1+\mathrm{z}\end{array}$ & $\mathrm{C} 22-\mathrm{H} 22 \mathrm{~A} \cdots \mathrm{O}$ - & $2.65(2), 154$ & 7.564 & -20.3 & -9.1 & -77.2 & 48.8 & -57.9 \\
\hline & & $\mathrm{C} 22-\mathrm{H} 22 \mathrm{~A} \cdots \mathrm{N} 5 \mathrm{~B}$ & $2.60(2), 174$ & & & & & & \\
\hline & & $\operatorname{Cg} 2 \cdots \operatorname{Cg} 6$ & $3.9703(1)^{\mathrm{b}}$ & & & & & & \\
\hline & & $\mathrm{C} 22 \mathrm{~A}-\mathrm{H} 22 \mathrm{~A} \cdots \mathrm{Cg} 4$ & $2.71^{\mathrm{a}}$ & & & & & & \\
\hline & & $\mathrm{C} 22 \mathrm{~B}-\mathrm{H} 22 \mathrm{~B} \cdots \mathrm{Cg} 1$ & $2.86^{\mathrm{a}}$ & & & & & & \\
\hline & $1-x,-1-y, 1-z$ & $\mathrm{C} 6 \mathrm{~A}-\mathrm{H} 6 \mathrm{~A} 1 \cdots \mathrm{Cg} 6$ & $2.88^{\mathrm{a}}$ & 12.307 & -7.9 & -2.7 & -17.8 & 12.2 & -16.2 \\
\hline & $1-\mathrm{x},-\mathrm{y},-\mathrm{z}$ & $\begin{array}{l}\text { S8A-O9A } \cdots \text { Cg2, } \\
\text { S8A-010A } \cdots \text { Cg2 }\end{array}$ & $\begin{array}{l}3.8039^{c} \\
3.5353^{c}\end{array}$ & 6.086 & -2.1 & -4.8 & -30.9 & 8.7 & -29.1 \\
\hline & $\mathrm{x}, \mathrm{y}, \mathrm{z}$ & $\mathrm{S} 8 \mathrm{~A}-\mathrm{O} 9 \mathrm{~A} \cdots \mathrm{Cg} 4$ & $3.5839^{c}$ & 11.489 & -5.3 & -4.2 & -34.6 & 15.3 & -28.8 \\
\hline \multirow[t]{6}{*}{ SADHEM } & $-\mathrm{x}, 2-\mathrm{y},-\mathrm{z}$ & $\begin{array}{l}\mathrm{N} 2-\mathrm{H} 2 \cdots \mathrm{O} 2 \\
\mathrm{Cg} 2 \cdots \mathrm{Cg} 2\end{array}$ & $\begin{array}{l}2.180(2), 172 \\
3.6231(3)^{\mathrm{b}}\end{array}$ & 5.336 & -54.6 & -27.3 & -60.6 & 56.8 & -85.7 \\
\hline & $1+\mathrm{x}, 1+\mathrm{y}, \mathrm{z}$ & $\begin{array}{l}\mathrm{C} 6-\mathrm{H} 6 \cdots \mathrm{N} 3 \\
\mathrm{C} 11-\mathrm{H} 11 \cdots \mathrm{O} 1\end{array}$ & $\begin{array}{l}2.50(1), 148 \\
2.714(2), 126\end{array}$ & 11.748 & -11.2 & -4.6 & -10.4 & 14.5 & -11.6 \\
\hline & $\mathrm{x}, 1+\mathrm{y}, \mathrm{z}$ & $\mathrm{C} 14-\mathrm{H} 14 \cdots \mathrm{O} 3$ & $2.700(2), 168$ & 8.345 & -18.7 & -5.8 & -28.8 & 20.8 & -32.5 \\
\hline & $\mathrm{x}, 2.5-\mathrm{y},-1 / 2+\mathrm{z}$ & $\mathrm{C} 3-\mathrm{H} 3 \mathrm{~A} \cdots \mathrm{O} 4$ & $2.418(2), 161$ & 13.228 & -12.4 & -3.0 & -7.1 & 8.9 & -13.6 \\
\hline & $-\mathrm{x}, 3-\mathrm{y},-\mathrm{z}$ & $\mathrm{C} 3-\mathrm{H} 3 \mathrm{C} \cdots \mathrm{Cg} 1$ & $2.91^{\mathrm{a}}$ & 7.434 & -18.7 & -8.6 & -67.2 & 34.5 & -60.0 \\
\hline & $-1-\mathrm{x}, 1 / 2+\mathrm{y}, 1 / 2-\mathrm{z}$ & $\mathrm{C} 18-\mathrm{H} 18 \mathrm{~B} \cdots \mathrm{Cg} 1$ & $2.97^{\mathrm{a}}$ & 13.948 & -2.2 & -1.7 & -13.4 & 10.0 & -7.3 \\
\hline \multirow[t]{5}{*}{ OKEWOS } & $-\mathrm{x}, 1-\mathrm{y}, 1-\mathrm{z}$ & $\mathrm{N} 2-\mathrm{H} 2 \mathrm{~A} \cdots \mathrm{N} 1$ & $2.03(2), 166$ & 9.033 & -99.7 & -51.8 & -42.1 & 109.6 & -84.0 \\
\hline & $\mathrm{x},-1+\mathrm{y}, \mathrm{z}$ & $\mathrm{C} 11-\mathrm{H} 11 \cdots \mathrm{O} 3$ & 2.655(1), 143 & 6.355 & -13.1 & -8.7 & -46.3 & 28.0 & -40.1 \\
\hline & $-\mathrm{x},-\mathrm{y}, 1-\mathrm{z}$ & $\operatorname{Cg} 1 \cdots \operatorname{Cg} 1$ & $3.5565(2)^{\mathrm{b}}$ & 8.602 & -8.3 & -5.8 & -47.1 & 23.8 & -37.4 \\
\hline & $1 / 2-x, 1 / 2-y, 1-z$ & $\mathrm{Cg} 2 \cdots \mathrm{Cg} 3$ & $3.5640(2)^{\mathrm{b}}$ & 6.753 & -27.7 & -10.1 & -88.8 & 59.3 & -67.3 \\
\hline & $1 / 2-x, 1 / 2+y, 1.5-z$ & $\mathrm{C} 5-\mathrm{H} 5 \cdots \mathrm{Cg} 2$ & $2.98^{\mathrm{a}}$ & 10.881 & -3.0 & -4.1 & -37.2 & 21.3 & -22.9 \\
\hline \multirow[t]{3}{*}{ SODGID } & $1-\mathrm{x}, 1-\mathrm{y},-\mathrm{z}$ & $\begin{array}{l}\mathrm{N} 3-\mathrm{H} 31 \cdots \mathrm{N} 2 \\
\mathrm{Cg} 2 \cdots \mathrm{Cg} 2\end{array}$ & $\begin{array}{l}2.18(3), 177 \\
3.8799(1)^{\mathrm{b}}\end{array}$ & 6.948 & -95.1 & -46.8 & -69.1 & 107.3 & -103.7 \\
\hline & $\mathrm{x}, 1 / 2-\mathrm{y},-1 / 2+\mathrm{z}$ & 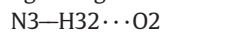 & $2.49(2), 174$ & 4.920 & -21.4 & -10.3 & -49.5 & 27.3 & -53.9 \\
\hline & $1-\mathrm{x}, 1 / 2+\mathrm{y}, 1 / 2-\mathrm{z}$ & $\mathrm{C} 15-\mathrm{H} 15 \cdots \mathrm{O} 1$ & $2.604(1), 160$ & 7.986 & -13.4 & -6.6 & -24.1 & 13.2 & -30.9 \\
\hline \multirow[t]{5}{*}{ CIKJAI } & $\mathrm{x}, \mathrm{y}, \mathrm{z}$ & 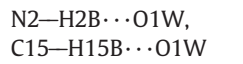 & $\begin{array}{l}1.913(3), 161 \\
2.657(3), 142\end{array}$ & 5.787 & -60.7 & -25.6 & -15.5 & 58.9 & -43.0 \\
\hline & $\mathrm{x},-1 / 2-\mathrm{y}, 1 / 2+\mathrm{z}$ & $\mathrm{O} 1 \mathrm{~W}-\mathrm{H} 1 \mathrm{~W} \cdots \mathrm{O} 4$ & $2.11(4), 155$ & 6.215 & -31.4 & -10.5 & -13.4 & 27.8 & -27.5 \\
\hline & $\mathrm{x}, 1 / 2-\mathrm{y}, 1 / 2+\mathrm{z}$ & $\mathrm{O} 1 \mathrm{~W}-\mathrm{H} 2 \mathrm{~W} \cdots \mathrm{O} 4$ & $2.16(4), 157$ & 4.749 & -19.7 & -8.3 & -9.8 & 19.8 & -18.0 \\
\hline & $\mathrm{x},-1 / 2-\mathrm{y},-1 / 2+\mathrm{z}$ & $\mathrm{C} 10-\mathrm{H} 10 \mathrm{~A} \cdots \mathrm{O} 3$ & 2.491(2), 132 & 7.999 & -14.3 & -4.0 & -14.2 & 8.6 & -24.0 \\
\hline & $\mathrm{x},-1+\mathrm{y}, \mathrm{z}$ & $\mathrm{S} 1-\mathrm{O} 2 \cdots \mathrm{Cg} 2$ & $3.143(1)^{\mathrm{c}}$ & 4.896 & -19.7 & -11.6 & -76.8 & 49.3 & -58.8 \\
\hline \multirow[t]{5}{*}{ CIKJIQ } & $\mathrm{x}, \mathrm{y}, \mathrm{z}$ & $\begin{array}{l}\text { N2-H2B } \cdots 01 \mathrm{~W} \\
\mathrm{C} 15-\mathrm{H} 15 \mathrm{~B} \cdots \mathrm{O} \text { W }\end{array}$ & $\begin{array}{l}1.920(2), 161 \\
2.668(3), 142\end{array}$ & 6.850 & -60.4 & -23.3 & -15.2 & 58.0 & -40.8 \\
\hline & $\mathrm{x},-1 / 2-\mathrm{y}, 1 / 2+\mathrm{z}$ & $\mathrm{O} 1 \mathrm{~W}-\mathrm{H} 1 \mathrm{~W} \cdots \mathrm{O} 4$ & $2.13(5), 148$ & 7.718 & -31.1 & -10.5 & -13.6 & 27.5 & -27.7 \\
\hline & $\mathrm{x}, 1 / 2-\mathrm{y}, 1 / 2+\mathrm{z}$ & $\mathrm{O} 1 \mathrm{~W}-\mathrm{H} 2 \mathrm{~W} \cdots \mathrm{O} 4$ & $2.31(4), 138$ & 5.851 & -10.1 & -4.9 & -9.0 & 12.1 & -12.0 \\
\hline & $\mathrm{x},-1 / 2-\mathrm{y},-1 / 2+\mathrm{z}$ & $\mathrm{C} 10-\mathrm{H} 10 \mathrm{~A} \cdots \mathrm{O} 3$ & $2.544(2), 129$ & 9.322 & -13.9 & -4.1 & -14.3 & 7.6 & -24.7 \\
\hline & $\mathrm{x},-1+\mathrm{y}, \mathrm{z}$ & $\mathrm{S} 1-\mathrm{O} 2 \cdots \mathrm{Cg} 2$ & $3.137(1)^{c}$ & 4.855 & -21.2 & -10.9 & -83.2 & 50.3 & -65.0 \\
\hline
\end{tabular}

a $\mathrm{H} \cdots$ Cg distance.

b Inter-centroid distance.

c $0 \cdots C$ g distance. 1: Cg1, Cg2 and Cg3 are the centroids of O3/N3/C7-C9, C1-C6 and C12-C17 rings, respectively; MULMA: Cg1, Cg2 and Cg3 are the centroids of O4-N3/C14-C16,

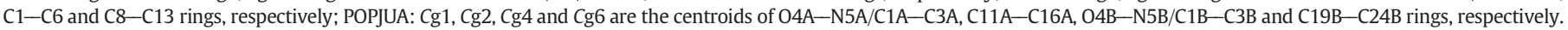

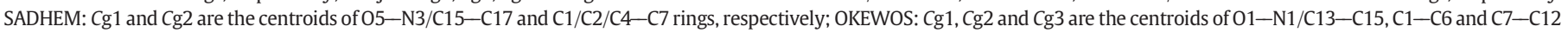
rings, respectively; SODGID: $\mathrm{Cg} 2$ is the centroid of $\mathrm{C} 10-\mathrm{C} 15$; CIKJAI and CIKJIQ: $\mathrm{Cg} 2$ is the centroid of C8-C13 ring

\subsection{Hirshfeld Surface Analysis}

In this work, we have calculated the Hirshfeld surfaces and 2D-fingerprint plots for investigating similarities and differences in the packing motifs of the series of eight structures under consideration. Fig. 3 show the molecular Hirshfeld $d_{\text {norm }}$ surfaces and full 2D-fingerprint plot of 1 and related compounds, respectively. The two near and larger red spots labeled 1 on the left in Fig. 3a are due to the formation of dimmers via $\mathrm{N} 2-\mathrm{H} 2 \cdots \mathrm{N} 3$ hydrogen bonds involving the acceptor N3 atom from the isoxazole ring. These contacts (motif I in Fig. 2) represent the stronger hydrogen bond interaction in the crystal, as reflected by the geometrical parameters and highest Pixel energy (Table 4). Another pair of smaller red areas labeled 1 at the center in Fig. $3 \mathrm{a}$ is also attributed to $\mathrm{H} \cdots \mathrm{N} / \mathrm{N} \cdots \mathrm{H}$ contacts, but representing $\mathrm{C}-\mathrm{H} \cdots \mathrm{N}$ hydrogen bonds between the $\mathrm{H} 14$ atom of the dimethylaminophenyl ring and the $\mathrm{N} 1$ atom of the azomethine group. In comparison to the former contact, the smaller extent of red area, the longer $\mathrm{H} \cdots \mathrm{N}$ distance of $2.51(2) \AA(2)$ and lower directionality $\left(157^{\circ}\right)$ in $\mathrm{C} 14-\mathrm{H} 14 \cdots \mathrm{N} 1$ 


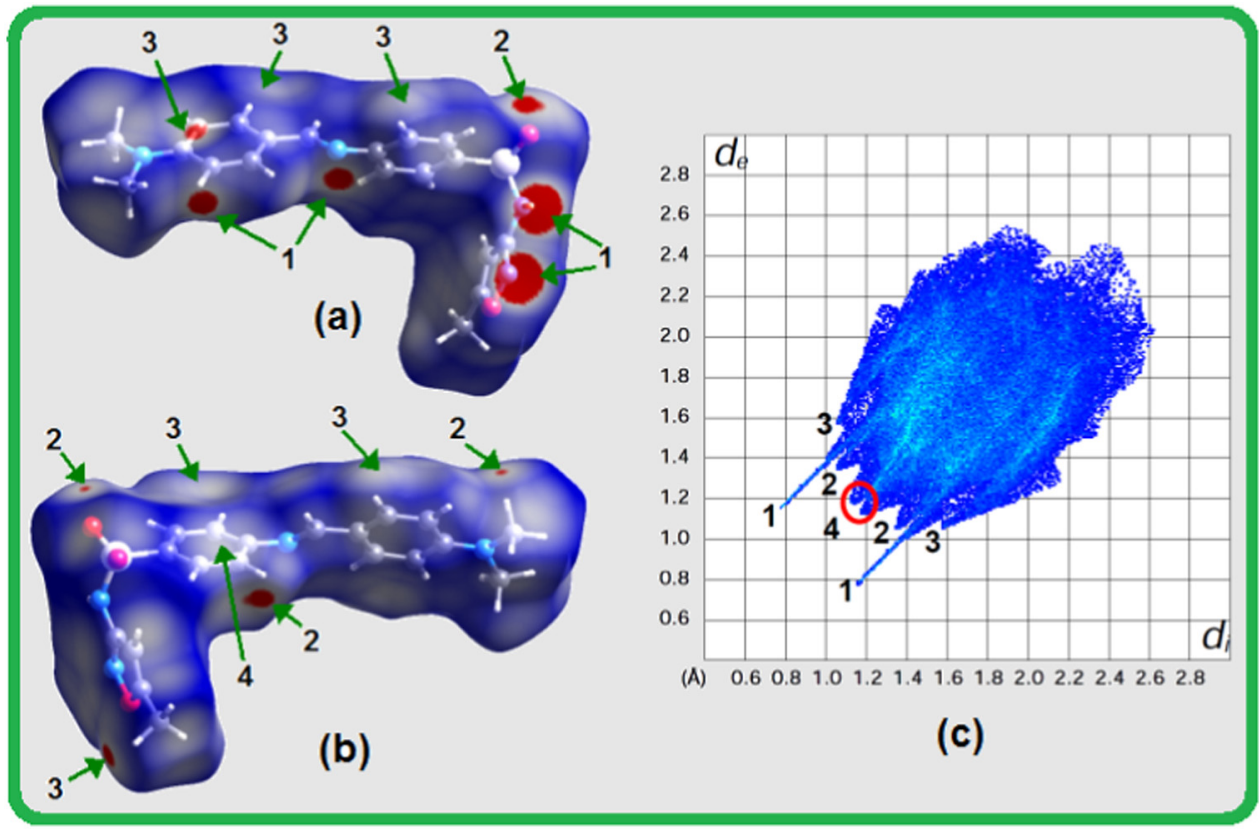

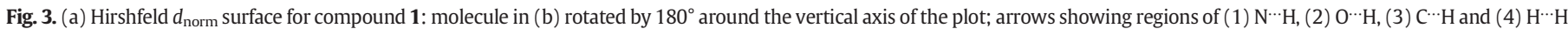

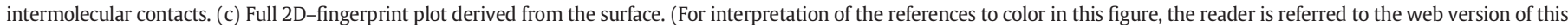
article.)

hydrogen bonds are indicative of a weaker interaction, supported by the lowest Pixel energy $\left(-22.7 \mathrm{~kJ} \mathrm{~mol}^{-1}\right)$ we found in this compound.

In Fig. 3b appears two small red spots (labeled 2 ) related to $\mathrm{H} \cdots \mathrm{O}$ / $\mathrm{O} \cdots \mathrm{H}$ contacts, which are associated to two different $\mathrm{C}-\mathrm{H} \cdots \mathrm{O}$ hydrogen bonds. The upper bright red region at right in Fig. 3a, and the pair of small red spots labeled 2 in Fig. 3b correspond to C18-H18A $\cdots$ O 1 hydrogen bonds, while a red spot at center in the rotated surface indicates C5-H5 $\cdots 01$ hydrogen bond. These contacts are highlighted in Fig. 2 as motifs III and IV, respectively, the former contributing $\left(-54.8 \mathrm{~kJ} \mathrm{~mol}^{-1}\right)$ $7.3 \mathrm{~kJ} \mathrm{~mol}^{-1}$ more stabilization towards the crystal packing than that of the motif IV. The regions highlighted as 3 correspond to $\mathrm{H} \cdots \mathrm{C} / \mathrm{C} \cdots \mathrm{H}$ contacts attributed to weak $\mathrm{C}-\mathrm{H} \cdots \pi$ interactions. The pair of white regions labeled 3 at the upper side in both the surfaces indicates $\mathrm{C} 17-\mathrm{H} 17 \cdot \cdots \mathrm{Cg} 2$ and $\mathrm{C} 3-\mathrm{H} 3 \cdots \mathrm{Cg} 3$ interactions, which are more visible in Fig. 3b. The bright red spot labeled 3 at left in Fig. 3a is related to a short $\mathrm{C} \cdot \mathrm{H}$ contact involving the benzylidene $\mathrm{C} 14$ carbon atom inside the surface, and the methyl $\mathrm{H} 10 \mathrm{C}$ hydrogen atom in a neighboring molecule (symmetry: $1 / 2+x, 1 / 2-y,-1 / 2+z$ ). The red spot labeled 3 at bottom left in Fig. 3b is the reciprocal H10C...C14 contact. This interaction is part of an insignificant $\mathrm{C} 10-\mathrm{H} 10 \mathrm{C} \cdot \mathrm{Cg} 3$ stacking with intermolecular distance longer than $3.0 \AA$. One of the several weak $\mathrm{H} \cdot \mathrm{H}$ contacts in this structure appears as a diffuse white region labeled 4 in Fig. 3b indicating a contact distance longer than the sum of van der Waals radii. Fig. S1 displays the $d_{\text {norm }}$ surfaces and decomposed 2D-fingerprint plots highlighting the main intermolecular contacts for all related compounds. Alam and co-workers have reported the synthesis and structural characterization of three hydrazones Schiff base analogues and the intermolecular interactions were evaluated by means the Hirshfeld surface analysis. This study reveals that these compounds were packed through $\mathrm{O}-\mathrm{H}, \mathrm{N}-\mathrm{H}, \mathrm{C}-\mathrm{H}$ and $\pi-\pi$ interactions [19].

The offset $\operatorname{Cg} 1 \cdots \operatorname{Cg} 1$ interactions we described in Section 3.1 for compound $\mathbf{1}$ can be seen on the Hirshfeld surface mapped with shape index (Fig. 4a), as a pattern of alternating red and blue triangles with suitable symmetry [40]. In addition, a relatively large and flat green region at the same side of the molecule on the corresponding curvedness surface (Fig. 4b) is also characteristic of $\pi$-stacking interactions [39]. A view of shape index surface in other orientation (Fig. 4c) clearly confirms the existence of the two $\mathrm{C}-\mathrm{H} \cdots \pi$ interactions described in
Section 3.1, as a pair of large and touching complementary triangles [40].

The pair of narrow pointed spikes labeled 1 around $\left(d_{e}+d_{i}\right)$ of $2.0 \AA$ in 2D-fingerprint plot (Fig. 3c) shows the presence of $\mathrm{N} \cdots \mathrm{H} / \mathrm{H} \cdots \mathrm{N}$ contacts, while a pair of short and broad wings labeled 2 around $\left(d_{e}+d_{i}\right)$ of $2.5 \AA$ is attributed to $0 \cdots \mathrm{H} / \mathrm{H} \cdots \mathrm{O}$ contacts. The $\mathrm{C} \cdots \mathrm{H} /$ $\mathrm{H} \cdots \mathrm{C}$ contacts appear in a characteristic way for $\mathrm{C}-\mathrm{H} \cdots \pi$ interactions, i.e. in the form of pronounced wings (labeled 3 ) on the sides of the plot with the shortest $d i+d e \cong 2.7-2.8 \AA$ [59]. The $\mathrm{H} \cdot \cdots \mathrm{H}$ interactions are highlighted in the middle of scattered points in 2D fingerprint map, with minimum values of $(d e+d i)$ around $2.3 \AA$.

The relative contributions to the Hirshfeld surface area due to the main intermolecular interactions for compound $\mathbf{1}$ and related structures are shown in Fig. 5. The calculations reveal that in all eight compounds, the intermolecular $\mathrm{H} \cdots \mathrm{H}$ contacts have a major contribution to the crystal packing in the wide range of $16 \%-44 \%$, as result of the differences in the relative content of $\mathrm{H}$-atoms. The $\mathrm{N} \cdots \mathrm{H} / \mathrm{H} \cdots \mathrm{N}$ contacts in $\mathbf{1}$ comprise $11.5 \%$ of total Hirshfeld surface area, slightly higher than those for MULMA, POPJUA, SADHEM, OKEWOS and SODGIG structures (8\%-10\%), In contrast, the decomposition of the fingerprint plot shows that $\mathrm{O} \cdots \mathrm{H} /$ $\mathrm{H}$. O O contacts comprise $17.9 \%$ of the total Hirshfeld surface area for the molecule of $\mathbf{1}$, slightly lower than those for remaining compounds (21\%-28\%). The contribution of $22.9 \%$ for $\mathrm{C} \cdot \mathrm{H} / \mathrm{H} \cdots \mathrm{C}$ contacts is relevant in $\mathbf{1}$ and similar in MULMA (24\%) and SODGID (25\%), corresponding to all intermolecular $\mathrm{C}-\mathrm{H} \cdots \mathrm{C}$ interactions of which $\mathrm{C}-\mathrm{H} \cdots \pi$ were clearly displayed in shape index and curvedness surfaces (Fig. 4), and 2D-fingerprint plot (Fig. 3c).

The presence of $C \cdot$. C contacts in the crystal structures is represented in fingerprint plots as areas of pale blue/green color on the diagonals at around $d i=d e \cong 1.7-1.8 \AA$, and correspond to the presence of $\pi \cdots \pi$ stacking interactions [40]. This feature can be observed in the decomposed 2D-fingerprint plots of most related structures (Fig. S1, column 4) where the C..C contacts (labeled 6) comprise from $2 \%$ to $6 \%$ of total Hirshfeld surface area. In the case of compound 1, we do not observe the characteristic areas of $\pi \cdots \pi$ stacking in fingerprint plot due to the relative percentage of $C \cdots C$ contacts is extremely low $(0.4 \%)$. In similar structures, the $\pi \cdots \pi$ stacks occur between benzene and/or benzylidene rings (Table 3) favoring a higher 


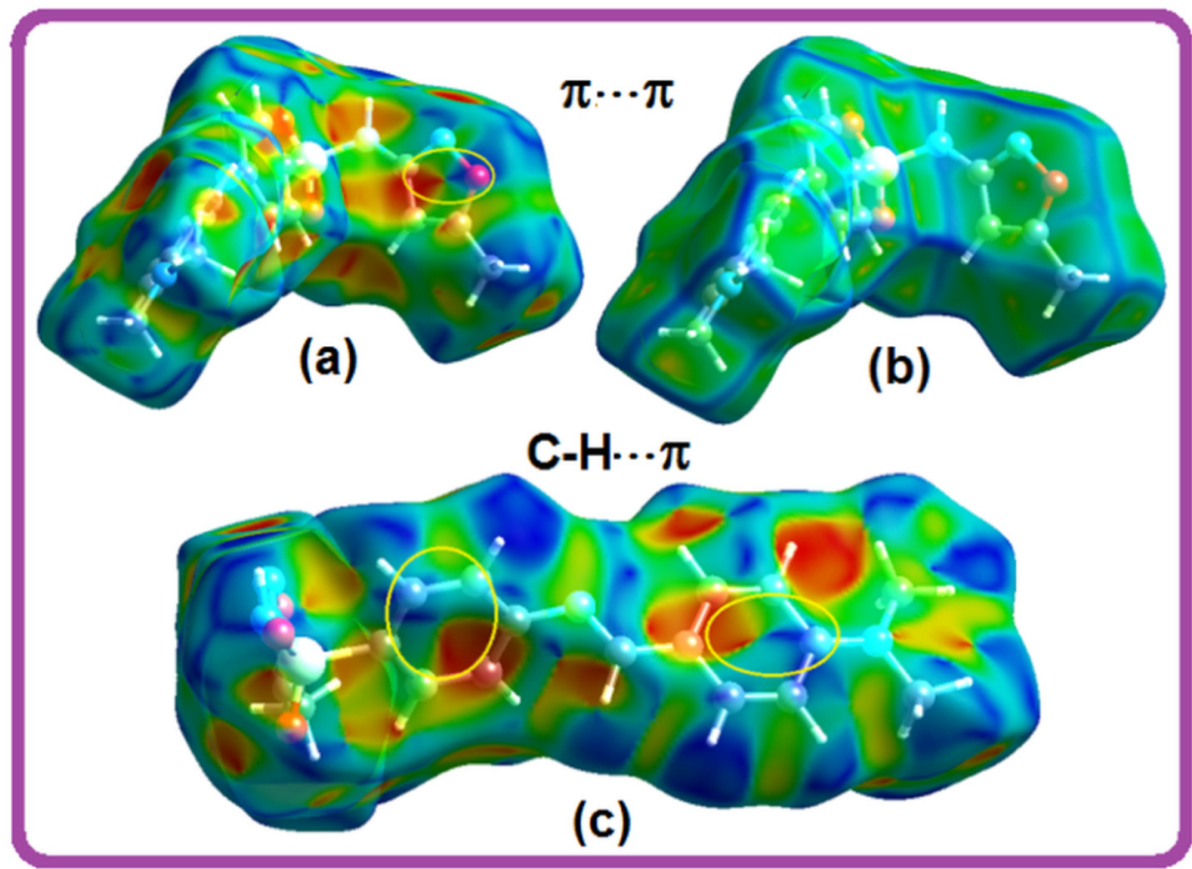

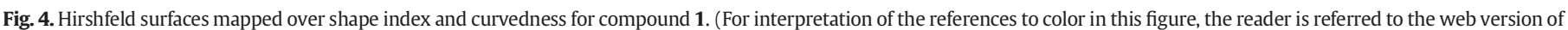
this article.)

contribution of $C \cdots C$ contacts than that for compound $\mathbf{1}$ where the offset $\mathrm{Cg} 1 \cdots \mathrm{Cg} 1$ interactions involve isoxazole rings fewer enriched of carbon atoms.

Another interesting feature in most of structures (except SODGID) is the presence of $\mathrm{O} \cdots \mathrm{C} / \mathrm{C} \cdots \mathrm{O}$ contacts (labeled 5 ) with contributions in the range $2 \%-7 \%$, corresponding to all intermolecular S-O $\cdots$ C interactions of which $\mathrm{S}-\mathrm{O} \cdots \pi$ were described in Section 3.1 as lone-pair $\cdots \pi$ contacts for POPJUA, CIKJAI and CIKJIQ structures. The lp $\cdots \pi$ contacts are identified in the Fig. S1, for monohydrate compounds.

To get a complete understanding towards the dominant electrostatic nature of $\mathrm{N}-\mathrm{H} \cdots \mathrm{N}$ and $\mathrm{N}-\mathrm{H} \cdots \mathrm{O}$ interactions, electrostatic potentials (ESP) were calculated over the Hirshfeld surfaces of all eight structures. Fig. S2 depicts the decomposed ESP map of compound $\mathbf{1}$ showing the $\mathrm{H} \cdots \mathrm{N} / \mathrm{N} \cdots \mathrm{H}$ contacts, while ESP values around $\mathrm{H}$-atoms and acceptor atoms $(\mathrm{N}, \mathrm{O})$ in hydrogen bonds interactions with dominant coulombic energy for related structures are listed in Table S1. The strongly electropositive blue region on the $\mathrm{H} 2$ atom (0.159 a.u.) in compound 1 interacts with the strongly electronegative red region over the $\mathrm{N} 3$ atom
( -0.093 a.u.) resulting in the formation of a dimer through strong $\mathrm{N} 2-\mathrm{H} 2 \cdots \mathrm{N} 3$ hydrogen bonds, and reflecting the substantial coulombic contribution of 53\% found for this motif in the PIXEL calculations. In comparison, the ESP is moderately positive around the $\mathrm{H} 14$ atom ( 0.053 a.u.) but much lower than that over the $\mathrm{H} 2$ atom, and less electronegative $(-0.081 \mathrm{au})$ about $\mathrm{N} 1$ atom leading to the formation of weak $\mathrm{C} 14-\mathrm{H} 14 \cdots \mathrm{N} 1$ hydrogen bonds with $26 \%$ from coulombic component towards the total cohesive energy.

Like in structure 1, the strongly electropositive potentials (0.163-0.183 a.u.) on the H-atoms interact with strongly electronegative potentials (from -0.082 to -0.097 a.u.) near the $\mathrm{N}$-atoms resulting in the occurrence of strongest $\mathrm{N}-\mathrm{H} \cdots \mathrm{N}$ hydrogen bonds for MULMA, POPJUA, OKEWOS and SODGID (Fig. S2, ESI†) with dominant coulombic contributions (45\%-53\%). We observe a similar behavior around hydrogen and oxygen atoms in $\mathrm{N}-\mathrm{H} \cdots \mathrm{O}$ hydrogen bonds for CIKJAI and CIKJIQ with even higher electropositive ( 0.218 and 0.217 a.u.) and electronegative $(-0.095$ and -0.104 a.u.) values, in agreement with higher coulombic percentages of $60 \%$ and $61 \%$, respectively.

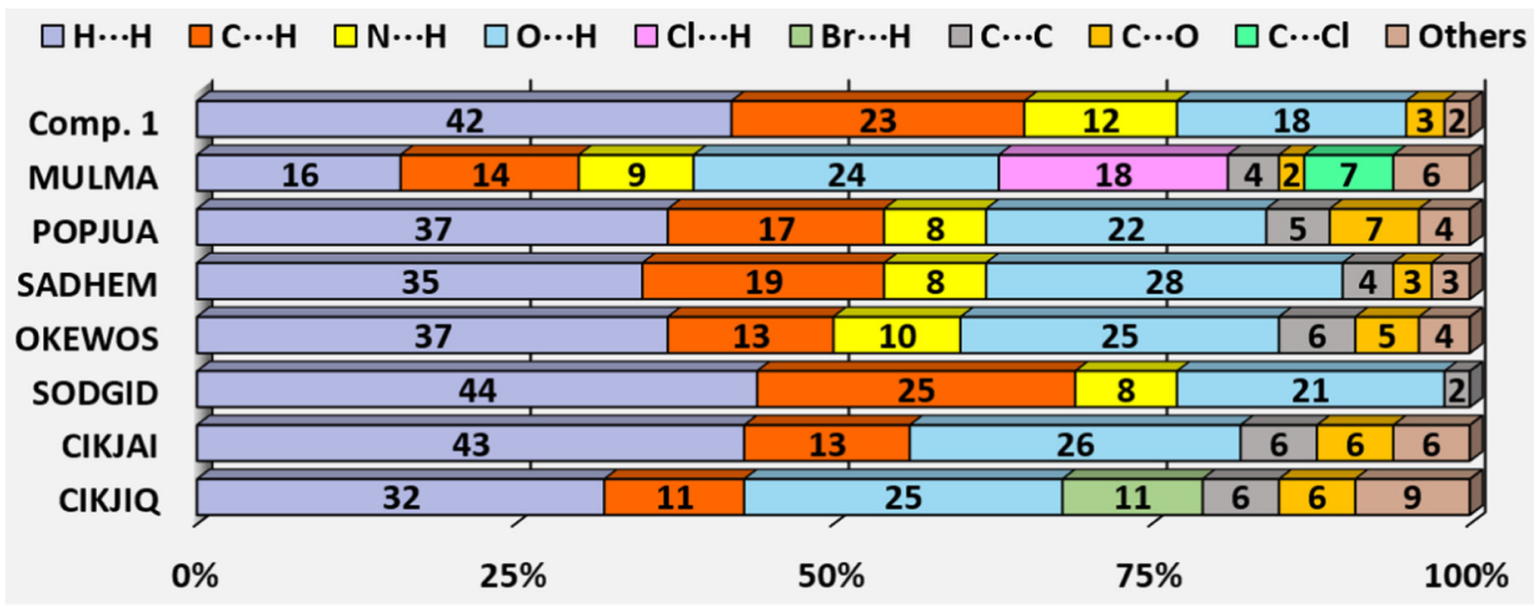

Fig. 5. Relative contributions of various intermolecular contacts to the Hirshfeld surface area in compound $\mathbf{1}$ and related structures. 


\subsection{NBO Analysis}

In order to evaluate energetically hyperconjugative and conjugative interactions in compound $\mathbf{1}$, the Natural Bond Orbital (NBO) analysis was carried out. The donor-acceptor interactions lead electron density delocalization associated with the occupancy shifts from the occupied Lewis-type (bonding or lone pair) NBO's to the unoccupied non-Lewis type (anti-bonding) NBO's of the molecule. The stabilization energy $E$ (2) associated with donor (i) and acceptor ( $\mathrm{j}$ ) delocalization is estimated from the second-order perturbation approach [60]:

$E(2)=\Delta E_{i j}=-q_{i} \frac{F_{i, j}^{2}}{E_{j}-E_{i}}$

where $q_{i}$ is the donor orbital occupancy, $E_{i}$ and $E_{j}$ are diagonal elements (orbital energies) of the donor and acceptor NBO's, respectively and $F_{i, j}$ is the off-diagonal Fock matrix element. The NBO calculations were performed at B3LYP/6-311 + $+\mathrm{G}(\mathrm{d}, \mathrm{p})$ level of approximation. The large value of $E(2)$ is an indicative of more intense interaction between electron donors and electron acceptors.

The $\pi$ electron delocalization produces $\pi$-conjugation or resonance owing to the $\pi \rightarrow \pi^{*}$ interactions. The overlap of various types of orbitals such as $\sigma \rightarrow \pi^{*}, \pi \rightarrow \sigma^{*}, \mathrm{lp} \rightarrow \pi^{*}$ and $\mathrm{lp} \rightarrow \sigma^{*}$ produces primary hyperconjugative interactions, whereas the orbital overlap $\sigma \rightarrow \sigma^{*}$ produces secondary hyper-conjugative interactions. The most relevant hyperconjugative interactions calculated by NBO analysis for the Schiff base BA-SMX are presented in Table 5. The results show $\pi \mathrm{C} 14-\mathrm{C} 15 \rightarrow \pi^{*}$ $\mathrm{C} 12-\mathrm{C} 13, \pi \mathrm{C} 12-\mathrm{C} 13 \rightarrow \pi^{*} \mathrm{C} 16-\mathrm{C} 17, \pi \mathrm{C} 3-\mathrm{C} 4 \rightarrow \pi^{*} \mathrm{C} 1-\mathrm{C} 2, \pi$ $\mathrm{C} 1-\mathrm{C} 2 \rightarrow \pi^{*} \mathrm{C} 3-\mathrm{C} 4$ and $\pi \mathrm{C} 8-\mathrm{C} 9 \rightarrow \pi^{*} \mathrm{C} 7-\mathrm{N} 3$ intramolecular interactions with energy values of 26.47, 21.76, 27.20, 35.79 and $27.44 \mathrm{kcal} \mathrm{mol}^{-1}$, respectively. These interactions are related to the resonance in the molecule and lead to intramolecular charge transfer causing stabilization of the molecular system. The lone pair of $\mathrm{O} 2$ oxygen donates its electrons to the $\sigma$-type anti-bonding orbitals $\sigma^{*} \mathrm{C} 1-\mathrm{S}, \sigma^{*}$ $\mathrm{S}-\mathrm{N} 2$ and $\sigma^{*} \mathrm{~S}-\mathrm{O} 1$ with stabilization energy of $18.88,16.76$ and $24.13 \mathrm{kcal} \mathrm{mol}^{-1}$, respectively. The interaction between lp N4 and the $\pi^{*}$ C14-C15 gives more stabilization of the molecule with $47.97 \mathrm{kcal} \mathrm{mol}^{-1}$ and is indicative of a double-bond character of the

Table 5

Second-order perturbation theory analysis of the Fock matrix for the Schiff base BA-SMX calculated at B3LYP/6-311++G(d,p) level.

\begin{tabular}{|c|c|}
\hline Interaction (donor $\rightarrow$ acceptor) ${ }^{a}$ & $E(2)^{\mathrm{b}}$ \\
\hline$\pi \mathrm{C} 14-\mathrm{C} 15 \rightarrow \pi^{*} \mathrm{C} 12-\mathrm{C} 13$ & 26.47 \\
\hline$\pi \mathrm{C} 14-\mathrm{C} 15 \rightarrow \pi^{*} \mathrm{C} 16-\mathrm{C} 17$ & 13.51 \\
\hline$\pi \mathrm{C} 12-\mathrm{C} 13 \rightarrow \pi^{*} \mathrm{C} 14-\mathrm{C} 15$ & 16.85 \\
\hline$\pi \mathrm{C} 12-\mathrm{C} 13 \rightarrow \pi^{*} \mathrm{C} 16-\mathrm{C} 17$ & 21.79 \\
\hline$\pi \mathrm{C} 16-\mathrm{C} 17 \rightarrow \pi^{*} \mathrm{C} 14-\mathrm{C} 15$ & 21.24 \\
\hline$\pi \mathrm{C} 16-\mathrm{C} 17 \rightarrow \pi^{*} \mathrm{C} 12-\mathrm{C} 13$ & 14.41 \\
\hline$\pi \mathrm{C} 3-\mathrm{C} 4 \rightarrow \pi^{*} \mathrm{C} 5-\mathrm{C} 6$ & 16.38 \\
\hline$\pi \mathrm{C} 3-\mathrm{C} 4 \rightarrow \pi^{*} \mathrm{C} 1-\mathrm{C} 2$ & 27.20 \\
\hline$\pi \mathrm{C} 5-\mathrm{C} 6 \rightarrow \pi^{*} \mathrm{C} 4-\mathrm{C} 3$ & 21.16 \\
\hline$\pi \mathrm{C} 5-\mathrm{C} 6 \rightarrow \pi^{*} \mathrm{C} 1-\mathrm{C} 2$ & 17.39 \\
\hline$\pi \mathrm{C} 1-\mathrm{C} 2 \rightarrow \pi^{*} \mathrm{C} 4-\mathrm{C} 3$ & 35.79 \\
\hline$\pi \mathrm{C} 8-\mathrm{C} 9 \rightarrow \pi^{*} \mathrm{C} 7-\mathrm{N} 3$ & 27.44 \\
\hline$\pi \mathrm{C} 12-\mathrm{C} 13 \rightarrow \pi^{*} \mathrm{C} 11-\mathrm{N} 1$ & 22.22 \\
\hline $\operatorname{lp} \mathrm{O} 2 \rightarrow \sigma^{*} \mathrm{C} 1-\mathrm{S}$ & 18.88 \\
\hline $\mathrm{lp} \mathrm{O} 2 \rightarrow \sigma^{*} \mathrm{~S}-\mathrm{N} 2$ & 26.76 \\
\hline $\mathrm{lp} 02 \rightarrow \sigma^{*} \mathrm{~S}-\mathrm{O} 1$ & 24.13 \\
\hline $\mathrm{lp} 01 \rightarrow \sigma^{*} \mathrm{C} 1-\mathrm{S}$ & 19.15 \\
\hline $\mathrm{lp} 01 \rightarrow \sigma^{*} \mathrm{~S}-\mathrm{N} 2$ & 25.71 \\
\hline $\operatorname{lp} 01 \rightarrow \sigma^{*} \mathrm{~S}-\mathrm{O} 1$ & 24.71 \\
\hline lp N4 $\rightarrow \pi^{*} \mathrm{C} 14-\mathrm{C} 15$ & 47.97 \\
\hline lp N1 $\rightarrow \pi^{*} \mathrm{C} 11-\mathrm{H}$ & 12.84 \\
\hline lp N2 $\rightarrow \pi^{*}$ C7-N3 & 23.10 \\
\hline $\mathrm{lp} O 3 \rightarrow \pi^{*} \mathrm{C} 7-\mathrm{N} 3$ & 14.72 \\
\hline $\mathrm{lp} \mathrm{O} 3 \rightarrow \pi^{*}$ C8-C9 & 35.19 \\
\hline
\end{tabular}

a See Fig. 1 for atoms numbering scheme. lp indicates the lone pair on the specified atom.

b Energy values in $\mathrm{kcal} \mathrm{mol}^{-1}$.
$\mathrm{N} 4-\mathrm{C} 15$ bond. The hyper-conjugative interaction lp $\mathrm{O} 3 \rightarrow \pi^{*} \mathrm{C} 8-\mathrm{C} 9$ (35.19 $\left.\mathrm{kcal} \mathrm{mol}^{-1}\right)$ is responsible for the stabilization of R3-ring (N3-O3/C7-C9) in the molecule.

\subsection{Vibrational Study}

A tentative assignment of the main vibrational modes expected for BA-SMX is listed on Table S2. Fig. 6 depicts the experimental IR and Raman spectra of the title compound. The vibrational analysis of the compound in solid state was supported by quantum chemical calculations at B3LYP/6-311 + $+\mathrm{G}(\mathrm{d}, \mathrm{p})$ level of theory, in comparison with related compounds as well as on basis of potential energy distributions (PED). The simulated IR and Raman spectra compared with the experimental ones are presented in Figs. S3 and S4, respectively.

\subsubsection{Assignment of Bands}

3.5.1.1. $C=N$ Vibrations. The vibrational modes of the azomethine groups are highly sensitive to the degree of charge transfer between donor and acceptors, and therefore such stretching modes are of particular interest for spectroscopists and it is relevant in the synthesis of coordination compounds using Schiff bases as ligands. The $\mathrm{C}=\mathrm{N}$ stretching mode generally appears in the frequency range $1674-1649 \mathrm{~cm}^{-1}$ as strong bands because of the large change in dipole moment during this vibration. For the compound under study, the medium intensity band located at $1618 \mathrm{~cm}^{-1}$ in the IR spectrum (1621 $\mathrm{cm}^{-1}$ in Raman) is assigned to the $\mathrm{C}=\mathrm{N}$ stretching mode. This mode is calculated at $1625 \mathrm{~cm}^{-1}$ with $67 \%$ of PED contribution. Generally, this mode appears mixed with $\mathrm{C}-\mathrm{C}$ stretching modes of the aromatic rings.

3.5.1.2. $\mathrm{SO}_{2} \mathrm{NH}$ Vibrations. The bands corresponding to the asymmetric and symmetric $\mathrm{SO}_{2}$ stretching modes generally appear at around 1315 and $1160 \mathrm{~cm}^{-1}$, respectively [61]. The inclusion of unsaturated functional groups to the $\mathrm{SO}_{2}$ moiety has a little effect on the $\mathrm{SO}_{2}$ stretching vibration. Such stability of the $\mathrm{SO}_{2}$ frequencies with respect to the neighboring unsaturated groups is due to the fact that both $\mathrm{S}=0$ bonds are not coplanar with the adjacent unsaturated moiety. Hence, there is no conjugation effect between the $S=0$ bonds and the functional groups adjacent, and the $\nu\left(\mathrm{SO}_{2}\right)$ frequencies remain in a constant region [61].

The strong band located at $1315 \mathrm{~cm}^{-1}$ in the IR spectrum (1300 $\mathrm{cm}^{-1}$ in Raman) is assigned to the asymmetric $\mathrm{SO}_{2}$ stretching mode. The calculated value for this mode is predicted at $1328 \mathrm{~cm}^{-1}$ with a contribution of $38 \%$. These results are in agreement with the assignment reported by Di Santo et al. that reports this mode as a strong

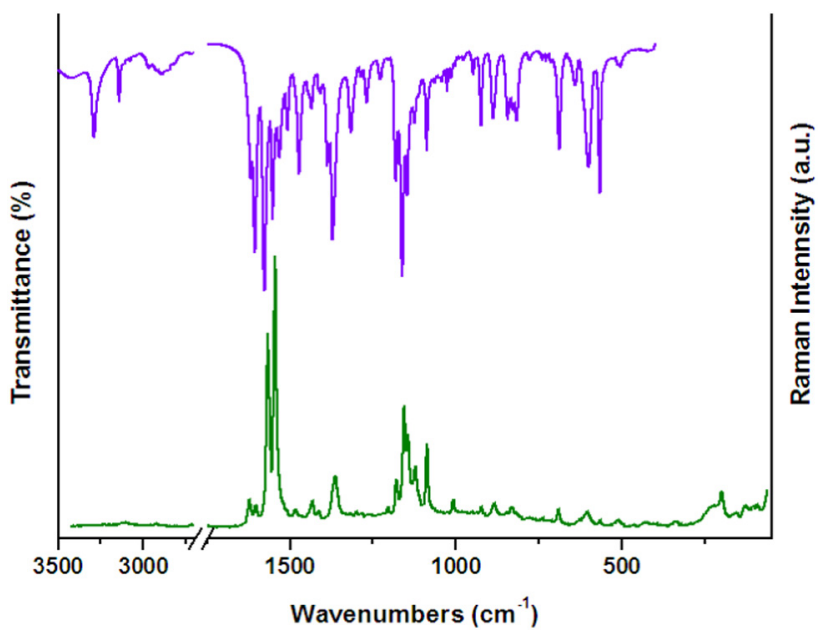

Fig. 6. Experimental IR and Raman spectra (solid) of the Schiff base BA-SMX. 
band in IR at $1314 \mathrm{~cm}^{-1}[62]$. For related Schiff bases this vibration was reported in the frequency range $1350-1300 \mathrm{~cm}^{-1}$ [63-65].

The IR spectra show a medium intensity band at $1147 \mathrm{~cm}^{-1}$ (1144 $\mathrm{cm}^{-1}$ in Raman), which can be assigned to symmetric $\mathrm{SO}_{2}$ stretching mode, in agreement with related compounds [62-66]. The calculated value for this mode is $1092 \mathrm{~cm}^{-1}$ with a PED contribution of $72 \%$.

The band corresponding to the $\mathrm{SO}_{2}$ in-plane bending mode and the wagging appear at 567 and $601 \mathrm{~cm}^{-1}$, respectively in the IR spectra with counterparts at 567 and $605 \mathrm{~cm}^{-1}$ in the Raman spectra.

The $\mathrm{S}-\mathrm{N}$ stretching vibrations appear in the spectral range 950$860 \mathrm{~cm}^{-1}[61,62,66,67]$. The IR spectra of the title compound show a medium intensity band located at $844 \mathrm{~cm}^{-1}$ which is tentative assigned to $\mathrm{S}-\mathrm{N}$ stretching mode, in agreement with the computed value of $831 \mathrm{~cm}^{-1}$ at B3LYP/6-311++G(d,p) approximation. The C-S stretching mode is tentatively assigned to the weak absorption observed at $690 \mathrm{~cm}^{-1}$ in the IR spectra $\left(690 \mathrm{~cm}^{-1}\right.$ in Raman), in good agreement with the computed value $\left(667 \mathrm{~cm}^{-1}\right)$ and with values reported in literature $[63,66]$.

The analysis of the IR and Raman spectra in the $\mathrm{N}-\mathrm{H}$ stretching mode region is of main interest for analyzing the presence of intra/intermolecular hydrogen bonds. For the compound under study, this mode is observed as a weak band in the IR spectra at $3289 \mathrm{~cm}^{-1}$. For its parent sulfamethoxazole, the $\mathrm{N}-\mathrm{H}$ stretching vibration appears as a strong band at $3467 \mathrm{~cm}^{-1}$ in the IR spectra [63]. From this comparison, it is apparent that the $\mathrm{N} 2-\mathrm{H} \cdots \mathrm{N} 3$ intermolecular hydrogen bonds are responsible for the red-shift and low intensity of the $\nu(\mathrm{N}-\mathrm{H})$ mode observed in the Schiff base.

3.5.1.3. $\mathrm{N}\left(\mathrm{CH}_{3}\right)_{2}$ Vibrations. For the assignment of methyl group modes, several fundamental vibrational modes can be associated to each $\mathrm{CH}_{3}$ group such as asymmetric and symmetric stretches, bends, rocking and torsional modes. The assignment of all these modes is shown in Table S2. The bands located at 2966 and $2927 \mathrm{~cm}^{-1}$ are assigned to the asymmetric $\mathrm{CH}_{3}$ stretching mode of the methyl groups attached to the amino $\mathrm{N}$ atom, in good agreement with the computed values (2933 and $2930 \mathrm{~cm}^{-1}$ ) and with results previously reported $[14,68]$. The weak band located at $1387 \mathrm{~cm}^{-1}$ in the IR spectra is assigned to the $\mathrm{CH}_{3}$ symmetric bending mode, with a computed value of $1396 \mathrm{~cm}^{-1}$ ( $83 \%$ of PED).

The IR spectrum shows two absorptions at 1227 and $949 \mathrm{~cm}^{-1}$, with low intensity, which are tentatively assigned to the $\mathrm{N}\left(\mathrm{CH}_{3}\right)_{2}$ asymmetric and symmetric stretching modes, respectively. Counterpart signal appears in the Raman spectrum at $952 \mathrm{~cm}^{-1}$. The computed values for these modes are predicted at 1228 and $935 \mathrm{~cm}^{-1}$, respectively.

The strong band located at $1371 \mathrm{~cm}^{-1}$ in the IR spectra $\left(1363 \mathrm{~cm}^{-1}\right.$ in Raman) is assigned to the $\mathrm{N} 4-\mathrm{C} 15(\mathrm{~N}-\mathrm{Ph})$ stretching mode coupled with $\mathrm{CH}_{3}$ rocking modes (See Table S2). The possible interaction between the lone pair N4 and the ring R1 indicates a double-bond character of the N4- 15 bond and could explain the shift to higher frequencies observed. The band located at $434 \mathrm{~cm}^{-1}$ in the Raman spectra is attributed to the $\mathrm{N}\left(\mathrm{CH}_{3}\right)_{2}$ in-plane bending mode.

3.5.1.4. Phenyl Ring Vibrations. The Schiff base studied in this work has two phenyl rings (R1 and R2) para-substituted, linked through an azomethine group. The weak band located at $3076 \mathrm{~cm}^{-1}$ in the IR spectrum is assigned to the $\mathrm{C}-\mathrm{H}$ stretching mode corresponding to R1. The weak bands at 3143 and $3112 \mathrm{~cm}^{-1}$ are assigned to the $\mathrm{C}-\mathrm{H}$ stretching mode of R2.

The ring $\mathrm{C}-\mathrm{C}$ stretching vibrations are prominent in the vibrational spectra of aromatic derivatives and generally these modes are influenced by substituents [61]. The strong bands observed in the IR spectrum at 1605 and $1552 \mathrm{~cm}^{-1}$ (1603 and $1544 \mathrm{~cm}^{-1}$ in Raman) are assigned to $\mathrm{C}-\mathrm{C}$ stretching modes corresponding to $\mathrm{R} 1$ and the bands located at 1575, 1382 and $1087 \mathrm{~cm}^{-1}$ in the IR spectra (1566 and $1087 \mathrm{~cm}^{-1}$ in Raman) could be tentatively assigned to $\mathrm{C}-\mathrm{C}$ stretching modes of R2. These results are in agreement with the computed values reported in Table S2.

The in-plane $\mathrm{C}-\mathrm{H}$ bending modes of the phenyl rings appear as a series of bands at $1532,1445,1162$ and $1124 \mathrm{~cm}^{-1}$ in the IR spectra and their counterparts in Raman are observed at 1483, 1155 and $1123 \mathrm{~cm}^{-1}$. These results are in agreement with calculations and PED analysis indicates that these modes have coupling with $\mathrm{C}-\mathrm{C}$ stretching modes of the phenyl ring and with other modes of substituents (See Table S2). The $\mathrm{C}-\mathrm{H}$ out-of-plane bending mode of $\mathrm{R} 2$ is assigned to the absorption observed at $829 \mathrm{~cm}^{-1}\left(831 \mathrm{~cm}^{-1}\right.$ in Raman), in good agreement with calculations.

3.5.1.5. Isoxazolyl Vibrations. It is known that the five-membered heterocycle ring modes cannot be interpreted as simple vibrational modes and most of them are of complex origin and involve different vibrational types. The medium intensity bands located at 1472 and $1269 \mathrm{~cm}^{-1}$ in the IR spectra are assigned to the $\mathrm{C}-\mathrm{N}$ and $\mathrm{C}-\mathrm{O}$ stretching modes, respectively. These frequencies values and the relative intensities are in very good agreement with the computed ones, as shown in Table S2. The IR spectrum shows a weak band located at $925 \mathrm{~cm}^{-1}\left(923 \mathrm{~cm}^{-1}\right.$ in Raman) and is assigned to the $\mathrm{N}-\mathrm{O}$ stretching mode with a $47 \%$ of PED contribution.

\section{6. ${ }^{1} \mathrm{H}$ and ${ }^{13} \mathrm{C}$ NMR Analysis}

The calculated ${ }^{1} \mathrm{H}$ and ${ }^{13} \mathrm{C}$ NMR chemical shifts $(\delta)$ have been performed with the GIAO method taking into account implicitly the solvent $\left(\mathrm{CHCl}_{3}\right)$. Experimental ${ }^{1} \mathrm{H}$ and ${ }^{13} \mathrm{C}$ NMR spectra of the Schiff base are shown in Figs. S5 and S6, respectively. Table 6 shows a comparison between experimental and calculated values. All data sets showed a linear relationship with R-square values for each spectrum above 0.999 (See Figs. S7 and S8).

The singlet located at $8.23 \mathrm{ppm}$ is assigned to the azomethine proton, in accordance with the values reported by similar structures [14]. In ${ }^{1} \mathrm{H}$ NMR spectra of BA-SMX, the absorption of aromatic ring protons is in the range 6.25-7.80 ppm, in good agreement with literature data [69]. The doublet located at $2.37 \mathrm{ppm}$ is assigned to the protons of the methyl group attached to isoxazole ring (R3) and the singlet at $3.07 \mathrm{ppm}$ is attributed to protons of methyl groups of $\mathrm{N}\left(\mathrm{CH}_{3}\right)_{2}$.

The peak observed at $162.2 \mathrm{ppm}$ in the ${ }^{13} \mathrm{C}$ NMR is assigned to the carbon atom of the azomethine group, in agreement with the literature values $[14,69]$ and calculations. The chemical shifts of the aromatic carbon atoms in the title compound appear in the range of 111.5$157.6 \mathrm{ppm}$. The expansion of the range of aromatic chemical shifts $(100-150 \mathrm{ppm})$ is attributed to the attachment of more than one electron withdrawing and electron realising substituents leading to deshielding of the protons and carbon atoms. The C10 attached to ring

Table 6

Comparison between experimental and theoretical ${ }^{1} \mathrm{H}$ and ${ }^{13} \mathrm{C}$ NMR chemical shifts (ppm) for the Schiff base BA-SMX.

\begin{tabular}{lllllll}
\hline & Experimental $^{\mathrm{a}}$ & $\mathrm{J}^{\mathrm{b}}$ & Calculated $^{\mathrm{c}}$ & & Experimental & Calculated \\
\hline $\mathrm{C} 9-\mathrm{CH}_{3}$ & $2.37(\mathrm{~d})$ & & 2.36 & $\mathrm{C} 10$ & 12.61 & 12.64 \\
$\mathrm{~N}-\mathrm{CH}_{3}$ & $3.07(\mathrm{~s})$ & & 3.06 & $\mathrm{C} 18, \mathrm{C} 19$ & 40.05 & 41.15 \\
$\mathrm{C} 8-\mathrm{H}$ & $6.25(\mathrm{q})$ & 0.7 & 6.57 & $\mathrm{C} 8$ & 85.53 & 99.41 \\
$\mathrm{C} 14-\mathrm{H}$ & $6.71(\mathrm{~d})$ & 9.0 & 6.78 & $\mathrm{C} 14, \mathrm{C} 16$ & 111.5 & 113.4 \\
$\mathrm{C} 5-\mathrm{H}$ & $7.18(\mathrm{~d})$ & 8.6 & 7.27 & $\mathrm{C} 3, \mathrm{C} 5$ & 121.7 & 125.1 \\
$\mathrm{C} 13-\mathrm{H}$ & $7.75(\mathrm{~d})$ & 9.0 & 7.75 & $\mathrm{C} 12$ & 124.1 & 128.9 \\
$\mathrm{C} 2-\mathrm{H}$ & $7.80(\mathrm{~d})$ & 8.6 & 7.95 & $\mathrm{C} 2, \mathrm{C} 6$ & 128.5 & 133.0 \\
$\mathrm{C} 11-\mathrm{H}$ & $8.23(\mathrm{~s})$ & & 8.42 & $\mathrm{C} 13, \mathrm{C} 17$ & 131.4 & 136.6 \\
& & & & $\mathrm{C} 1$ & 134.4 & 140.5 \\
& & & & $\mathrm{C} 15$ & 153.1 & 157.4 \\
& & & & $\mathrm{C} 4, \mathrm{C} 7$ & 157.6 & 165.5 \\
& & & & $\mathrm{C} 11$ & 162.2 & 167.2 \\
& & & $\mathrm{C} 9$ & 171.1 & 180.2 \\
\hline
\end{tabular}

\footnotetext{
a Multiplicity in parentheses; s: singlet, d: doublet, q: quartet.

b Coupling constant in $\mathrm{Hz}$.

Calculated at B3LYP/6-311 + G(2d,p) taking into account implicitly the solvent.
} 
R3 appears as a peak located at $12.61 \mathrm{ppm}$ and the carbon atoms of the methyl groups of $\mathrm{N}\left(\mathrm{CH}_{3}\right)_{2}$ are located at $40.05 \mathrm{ppm}$, in accordance with reported values [14]. As can be seen in Table 6 , the results obtained by quantum chemical calculations using the GIAO method are in reasonable agreement with the experimental values.

\subsection{Electronic Spectra}

The experimental and calculated electronic absorption spectra of the Schiff base BA-SMX are shown in Fig. 7. The experimental spectrum was measured in acetonitrile as solvent in the $200-800 \mathrm{~nm}$ spectral range. Table 7 shows the main absorption bands, correlated with the calculated values and a tentative assignment of the electronic transitions. Based on these results, there is a very good agreement between experimental and calculated electronic spectra.

As can be seen in Table 7, the calculated wavelengths are 387, 310 and $293 \mathrm{~nm}$. The electronic spectrum in acetonitrile presents bands located at $370 \mathrm{~nm}, 315 \mathrm{~nm}$ and $242 \mathrm{~nm}$. Taking into account the oscillator strengths, the band located at $370 \mathrm{~nm}$ in the experimental electronic spectra is assigned to an electronic transition from HOMO to LUMO molecular orbitals ( $\pi \rightarrow \pi^{*}$ nature) with $100 \%$ of contribution. The frontier molecular orbitals mainly involved in the electronic transitions are drawn in Fig. S9. The HOMO corresponds to a $\pi$ bonding system localized over both phenyl rings and $p$-type orbitals strongly located on the $\mathrm{N}$ atom of the azomethine group and over both nitrogen and oxygen of ring R3. The LUMO exhibits $\pi$ anti-bonding character delocalized over both phenyl rings and non-bonding character of $\mathrm{N}$ atom of the $\mathrm{N}\left(\mathrm{CH}_{3}\right)_{2}$ group. The energy difference between both HOMO and LUMO molecular orbitals is an important parameter in determining molecular electrical transport properties [70]. The energy gap HOMO-LUMO was predicted to be $2.430 \mathrm{eV}$ obtained at B3LYP/6-311 + $+\mathrm{G}(\mathrm{d}, \mathrm{p})$ level, indicating that this Schiff base is very stable.

The shoulder observed at $315 \mathrm{~nm}$ is assigned to the HOMO$1 \rightarrow$ LUMO excitation, with minor contribution of HOMO $\rightarrow$ LUMO +1 transition. The band observed at $242 \mathrm{~nm}$ in the experimental electronic spectrum is assigned to HOMO $\rightarrow$ LUMO +1 (65\%) and HOMO $\rightarrow$ LUMO +2 (29\%) transitions, both of $\pi \rightarrow \pi^{*}$ nature.

\section{Conclusions}

A new Schiff base, namely 4-(4-dimethylaminobenzylideneamino)$\mathrm{N}$-(5-methyl-3-isoxazolyl)benzenesulfonamide (1), was synthesized, characterized by spectral studies (IR, Raman, ${ }^{1} \mathrm{H}$ NMR, ${ }^{13} \mathrm{C}$ NMR and electronic absorption) and its crystal structure elucidated by X-ray

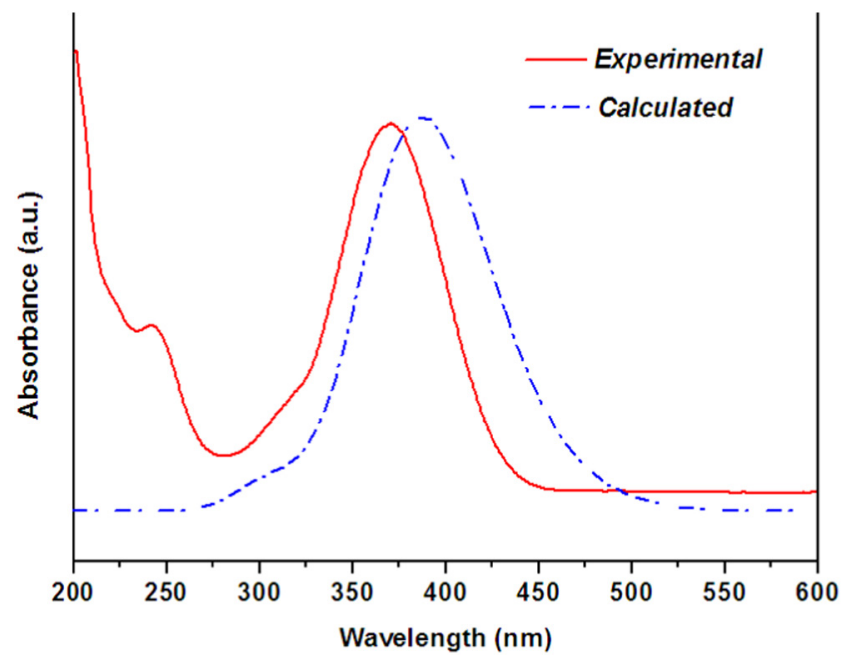

Fig. 7. Experimental and calculated electronic spectra for the Schiff base BA-SMX in acetonitrile as solvent.
Table 7

Calculated and experimental wavelengths ( $\mathrm{nm}$ ), oscillator strengths and tentative assignments of the main electronic transitions in the Schiff base BA-SMX.

\begin{tabular}{|c|c|c|c|}
\hline \multicolumn{2}{|c|}{ Wavelength (nm) } & \multirow[t]{2}{*}{ Oscillator strength $(f)^{\mathrm{b}}$} & \multirow[t]{2}{*}{ Tentative assignment $^{\mathrm{c}}$} \\
\hline Calculated $^{\mathrm{a}}$ & Experimental & & \\
\hline 387 & 370 & 1.1730 & HOMO $\rightarrow$ LUMO (100\%) \\
\hline 310 & 315 & 0.0996 & $\begin{array}{l}\text { HOMO-1 } \rightarrow \text { LUMO }(78 \%) \\
\text { HOMO } \rightarrow \text { LUMO + } 1(13 \%)\end{array}$ \\
\hline 293 & 242 & 0.0038 & $\begin{array}{l}\text { HOMO } \rightarrow \text { LUMO + } 1(65 \%) \\
\text { HOMO } \rightarrow \text { LUMO + } 2(29 \%)\end{array}$ \\
\hline
\end{tabular}

a Calculated at B3LYP/6-311++G(d,p) approximation taking into account implicitly solvent.

b Oscillator strengths of calculated transitions are in atomic units.

c Major contributions are shown in parentheses.

diffraction methods. The molecular conformation has been corroborated by comparison of geometric parameters between the DFT optimized and X-ray determined structures. Intermolecular interactions in title compound and seven related structures were studied with Pixel energies and Hirshfeld surfaces showing that the crystal packing is stabilized by strong $\mathrm{N}-\mathrm{H} \cdots \mathrm{N}$ or $\mathrm{N}-\mathrm{H} \cdots \mathrm{O}$ hydrogen bonds and weaker $\mathrm{C}-\mathrm{H} \cdots \mathrm{O}$ hydrogen bonds, together with short $\pi \cdots \pi$ and/or $\mathrm{C}-\mathrm{H} \cdots \pi$ stacking contacts for six structures. The presence of rare lp $\cdots \pi$ stacks was observed only in three compounds. Similarities and differences we found in the formation of diverse intermolecular patterns revealed that the packing of the molecules is sensitive towards the nature of substituents on the sulfonamide and benzylidene groups. Pixel energy calculations indicated a dominant coulombic contribution towards the total cohesive energy for $\mathrm{N}-\mathrm{H} \cdots \mathrm{N}$ and $\mathrm{N}-\mathrm{H} \cdots \mathrm{O}$ hydrogen bonds. Hirshfeld surface analysis has shown that the close contacts in all structures are dominated mainly by $\mathrm{H} \cdot \mathrm{H}$ contacts, indicating that van der Waals forces exert a crucial effect on the stabilization of the packing. 2D-fingerprint plots enabled quantifying the contribution of specific contacts to the Hirshfeld surface area. The electrostatic potentials mapped on the Hirshfeld surfaces confirmed the electrostatic nature of the strong hydrogen bonds according to the energetic results. This work represents the first close insight on intermolecular interactions into a series of Schiff base sulfonamide derivatives, providing a set of tools for understanding of crystal packing in this type of compounds.

\section{Acknowledgements}

The authors thank for financial support to SCAIT (D542/2), CONICET (PIP 11220130100651CO and PIP 0205), ANPCyT (PICT 2013-0697), and of Argentina. F.F.S. thanks Consejo Interuniversitario Nacional (CIN) for a fellowship.

\section{Appendix A. Supplementary data.}

Supplementary data to this article can be found online at http://dx. doi.org/10.1016/j.saa.2017.05.066.

\section{References}

[1] K. Isik, F. Özdemir-Kocak, Microbiol. Res. 164 (2009) 49-58.

[2] Z.H. Chohan, Mahmood-Ul-Hassan, M. Khan, C.T. Supuran, J. Enzyme Inhib. Med. Chem. 20 (2005) 183.

[3] Z. Huang, Z. Lin, J. Huang, Eur. J. Med. Chem. 36 (2001) 863.

[4] J.C. Medina, D. Roche, B. Shan, R.M. Learned, W.P. Frankmoelle, D.L. Clark, Bioorg. Med. Chem. Lett. 9 (1999) 1843.

[5] T. Owa, H. Yoshino, T. Okauchi, K. Yoshimatsu, Y. Ozawa, N. HataSugi, T. Nagasu, N. Koyanagi, K. Kiyoh, J. Med. Chem. 42 (1999) 3789.

[6] R.D. Jones, D.A. Summerville, F. Basolo, Chem. Rev. 79 (1979) 139-179.

[7] P. Przybylski, A. Huczynski, K. Pyta, B. Brzezinski, F. Barti, Curr. Org. Chem. 13 (2009) 124.

[8] M. Hranjec, K. Starcevic, S.K. Pavelic, P. Lucin, K. Pavelik, G.K. Zamola, Eur. J. Med. Chem. 46 (2011) 2274-2279.

[9] A. McAuiffe, R.V. Parish, S.M. Abu-El-Wafa, R.M. Issa, Inorg. Chim. Acta 115 (1986) 91-94.

[10] J.L. Sessler, P.J. Melfi, G. Dan Pantos, Coord. Chem. Rev. 250 (2006) 816-843. 
[11] S. Destri, I.A. Khotina, W. Porzio, Macromolecules 31 (1998) 1079-1086.

[12] M.I. Khan, A. Khan, I. Hussain, M.A. Khan, S. Gul, M. Iqbal, I. Ur-Rahman, F. Khuda, Inorg. Chem. Commun. 35 (2013) 104-109.

[13] S. Mondal, S.M. Mandal, T.K. Mondal, C. Sinha, Spectrochim. Acta A 150 (2015) 268-279.

[14] M. Rocha, A. Di Santo, G.A. Echeverría, O.E. Piro, F.D. Cukiernik, S.E. Ulic, D.M. Gil, J. Mol. Struct. 1133 (2017) 24-36.

[15] J.D. Dunitz, A. Gavezzotti, Chem. Soc. Rev. 38 (2009) 2622-2633.

[16] A. Gavezzotti, New J. Chem. 35 (2011) 1360-1368.

[17] S.K. Seth, I. Saha, C. Estarellas, A. Frontera, T. Kar, S. Mukhopadhyay, Cryst. Growth Des. 11 (2011) 3250-3265.

[18] A. Saeed, M. Bolte, M.F. Erben, H. Pérez, CrystEngComm 17 (2015) 7551-7563.

[19] M.S. Alam, D.U. Lee, Spectrochim. Acta A 145 (2015) 563-574.

[20] A. Saaed, U. Flörke, A. Fantoni, A. Khurshid, H. Pérez, M. Erben, CrystEngComm 19 (2017) 1495-1508.

[21] A. Di Santo, G.A. Echeverría, O.E. Piro, H. Pérez, A. Ben Altabef, D.M. Gil, J. Mol. Struct. 1134 (2017) 492-503.

[22] M. Owczarek, I. Majerz, R. Jakubas, CrystEngComm 16 (2014) 7638-7648.

[23] N. Shamsudin, A.L. Tan, D.J. Young, M.M. Jotani, A. Otero-de-la-Rozad, E.R.T. Tiekink, Acta Cryst E72 (2016) 563-569.

[24] M.A. Spackman, J.J. McKinnon, D. Jayatilaka, CrystEngComm 10 (2008) 377-388.

[25] CrysAlisPro, Oxford Diffraction Ltd., Version 1.171.37.31, 2014 (release 14-01-2014 CrysAlis171 .NET).

[26] G.M. Sheldrick, Acta Crystallogr. A64 (2008) 112-122.

[27] A. Spek, Acta Crystallogr. D65 (2009) 148-155.

[28] C.F. Macrae, I.J. Bruno, J.A. Chisholm, P.R. Edgington, P. McCabe, E. Pidcock, L. Rodriguez-Monge, R. Taylor, J. van de Streek, P.A. Wood, J. Appl. Crystallogr. 41 (2008) 466-470.

[29] M.J. Frisch, J.A. Pople, J.S. Binkley, J. Chem. Phys. 80 (1984) 3265;

M.J. Frisch, G.W. Trucks, H.B. Schlegel, G.E. Scuseria, M.A. Robb, J.R. Cheeseman, J.A Montgomery Jr., T. Vreven, K.N. Kudin, J.C. Burant, J.M. Millam, S.S. Iyengar, J Tomasi, V. Barone, B. Mennucci, M. Cossi, G. Scalmani, N. Rega, G.A. Petersson, H. Nakatsuji, M. Hada, M. Ehara, K. Toyota, R. Fukuda, J. Hasegawa, M. Ishida, T. Nakajima, Y. Honda, O. Kitao, H. Nakai, M. Klene, X. Li, J.E. Knox, H.P. Hratchian, J.B. Cross, C. Adamo, J. Jaramillo, R. Gomperts, R.E. Stratmann, O. Yazyev, A.J. Austin, R Cammi, C. Pomelli, J.W. Ochterski, P.Y. Ayala, K. Morokuma, G.A. Voth, P. Salvador, J.J. Dannenberg, V.G. Zakrzewski, S. Dapprich, A.D. Daniels, M.C. Strain, O. Farkas, D.K. Malick, A.D. Rabuck, K. Raghavachari, J.B. Foresman, J.V. Ortiz, Q. Cui, A.G. Baboul, S. Clifford, J. Cioslowski, B.B. Stefanov, G. Liu, A. Liashenko, P. Piskorz, I. Komaromi, R.L. Martin, D.J. Fox, T. Keith, M.A. Al-Laham, C.Y. Peng, A. Nanayakkara, M. Challacombe, P.M.W. Gill, B. Johnson, W. Chen, M.W. Wong, C. González, J.A. Pople, Gaussian 03, revision C.02, Gaussian Inc., Wallingford, CT, 2004.

[30] A.D. Becke, J. Chem. Phys. 98 (1993) 5648

[31] C. Lee, W. Yang, R.G. Parr, Phys. Rev. B 37 (1988) 785

[32] M.H. Jamróz, Spectrochim. Acta A 114 (2013) 220.

33] M.H. Jamróz, Vibrational Energy Distribution Analysis VEDA4,Warsaw, 2004

[34] P. Elliot, F. Furche, K. Burke, Ver. Comp. Chem. 26 (2009) 91.

[35] K. Wolinski, J.F. Hinton, P. Pulay, J. Am. Chem. Soc. 112 (1990) 8251-8260.

[36] A. Gavezzotti, J. Phys. Chem. B 107 (2003) 2344-2353.

[37] A. Gavezzotti, J. Phys. Chem. B 106 (2002) 4145-4154.
[38] J.J. McKinnon, D. Jayatilaka, M.A. Spackman, Chem. Commun. (2007) 3814-3816.

[39] M.A. Spackman, D. Jayatilaka, CrystEngComm 11 (2009) 19-32.

[40] J.J. McKinnon, M.A. Spackman, A.S. Mitchell, Acta Crystallogr. 60B (2004) 627-668.

[41] S.K. Wolf, D.J. Grimwood, J.J. McKinnon, M.J. Turner, D. Jayatilaka, M.A. Spackman, CrystalExplorer (version 3.1), University of Western Australia, 2012.

[42] I. Khan, P. Panini, S. Ud-Din Khan, U. Ali Rana, H. Andleeb, D. Chopra, S. Hameed, J. Simpson, Cryst. Growth Des. 16 (2016) 1371-1386.

[43] D. Jayatilaka, D.J. Grimwood, A. Lee, A. Lemay, A.J. Russel, C. Taylor, S.K. Wolff, P. Cassam-Chenai, A. Whitton, TONTO - A System for Computational Chemistry, 2005.

[44] L.J. Farrugia, J. Appl. Crystallogr. 30 (1997) 565.

[45] T.P. Singh, U. Patel, M. Haridas, Acta Crystallogr. C40 (1984) 2088-2091.

[46] V.L. Abramenko, V.S. Sergienko, Russ. J. Inorg. Chem. 47 (2002) 905-914.

[47] Y.M. Chumakox, V.I. Tsapkov, G. Bocelli, B.Y. Antosyak, Acta Crystallogr. C61 (2005) 460-463.

[48] A. Subashini, M. Hemamalini, P. Thomas Muthiah, G. Bocelli, A. Cantoni, J. Chem. Crystallogr. 39 (2009) 112-116.

[49] S.C. Nyburg, J. Cryst. Mol. Struct. 3 (1973) 331-336.

[50] M. Haridas, R.K. Tiwari, T.P. Singh, Acta Cryst 40C (1984) 658-660.

[51] M. Ghosh, A.A.K. Basak, S.K. Mazumdar, Acta Crystallogr. 47C (1991) 577-580.

[52] N. Sahu, D. Das, S. Mondal, S. Roy, P. Dutta, N. Sepay, S. Gupta, E. López-Torres, C. Sinha, New J. Chem. 40 (2016) 5019-5031.

[53] M. Yıldı, H. Ünver, D. Erdener, N.O. Iskeleli, J. Chem. Crystallogr. 40 (2010) 691-695.

[54] Y.M. Chumakov, V.I. Tsapkov, G. Bocelli, B.Y. Antosyak, Acta Crystallogr. 61C (2005) 0460-0463.

[55] D. Mustafa, T. Hasan, T. Tuncay, H. Tuncer, Acta Crystallogr. E70 (2014) o726-o727.

56] C. Janiak, J. Chem. Soc. Dalton Trans. (2000) 3885-3896.

[57] S.K. Seth, D. Sarkar, T. Kar, CrystEngComm 13 (2011) 4528-4535.

[58] S.K. Seth, CrystEngComm 15 (2013) 1772-1781.

[59] E. Rozycka-Sokolowska, B. Marciniak, J. Ławeck, B. Bujnicki, J. Drabowicz, A. Rykowski, J. Sulfur Chem. 34 (2013) 651-660.

[60] J.P. Foster, F. Weinhold, J. Am. Chem. Soc. 102 (1980) 7211-7218.

[61] D. Lin-Vien, N.B. Colthup, W.G. Fateley, J.G. Graselli, The Handbook of Infrared and Raman Characteristic Frequencies of Organic Molecules, Academic Press, Inc., 1991

[62] A. Di Santo, D.M. Gil, F. Pomiro, O.E. Piro, G.A. Eheverría, M. Arena, C. Luciardi, R.E. Carbonio, A. Ben Altabef, Inorg. Chim. Acta 436 (2015) 16-22.

[63] H. Ebrahimi, J.S. Hadi, H.S. Ansari, J. Mol. Struct. 1039 (2013) 37-45.

[64] R.C. Maurya, P. Patel, S. Rajput, Synth. React. Inorg. Met. Org. 33 (2003) 801-816.

[65] A. Chandran, Y. Shyma Mary, H.T. Varghese, C.Y. Panicker, P. Padzera, Spectrochim. Acta A 79 (2011) 1584-1592.

[66] D.M. Gil, O.E. Piro, G.A. Echeverría, M.E. Tuttolomondo, A. Ben Altabef, Spectrochim. Acta A 116 (2013) 122-131.

[67] A. Hangan, A. Bodoki, L. Oprean, G. Alzuet, M. Liu-González, J. Borrás, Polyhedron 29 (2010) 1305-1313.

[68] M. Rocha, A. Di Santo, J.M. Arias, D.M. Gil, A. Ben Altabef, Spectrochim. Acta A 136 (2015) 635-643.

[69] R. Silverstein, F. Webster, Spectrometric Identification of Organic Compounds, Wiley, 2006.

[70] V. Arjunan, L. Devi, R. Subbalakshmi, T. Rani, S. Mohan, Spectrochim. Acta A 130 (2014) 164 